M. Kita

Nagoya Math. J.

Vol. 135 (1994), 55-85

\title{
ON VANISHING OF THE TWISTED RATIONAL DE RHAM COHOMOLOGY ASSOCIATED WITH HYPERGEOMETRIC FUNCTIONS
}

\author{
MICHITAKE KITA
}

\section{Introduction}

Recent development in hypergeometric functions in several variables has made the importance of studying twisted rational de Rham cohomology clear to many specialists. Roughly speaking, a hypergeometric function in our sense is the integral of a product of complex powers of polynomials $P_{j}\left(u_{1}, \ldots, u_{n}\right): \int U d u_{1} \wedge$ $\cdots \wedge d u_{n}, U=\Pi P_{j}^{\alpha_{j}}$, integration being taken over some cycle. So we are led naturally to consider the twisted rational de Rham cohomology, which is a direct generalization of the usual de Rham cohomology to multivalued case. Thus it will be useful to give the exposition of twisted de Rham cohomology, which is necessary to the study of hypergeometric functions and this paper aims at the point. In the paper, we shall consider also the logarithmic complex, which is a very important subcomplex of the twisted rational de Rham complex and is quasiisomorphic to the complex in many good cases. We explain the content of the paper in more details; Let $D$ be the divisor of $\mathbf{C}^{n}$ defined by $\Pi P_{j}$ and set $\omega=d U / U$. In $\S 1$ and $\S 2$ we shall show some basic properties of logarithmic forms that are necessary to our later applications. In $\S 3$ we shall treat the case where each $P_{j}$ is homogeneous and show acyclicity of the twisted de Rham complex under the condition $\sum \alpha, \operatorname{deg} P_{j} \notin \mathbf{Z}$. To go further to inhomogeneous case, we introduce in $\S 4$ the degree frltration on the logarithmic complex and compare the associated graded complex with the complex $\left(\Omega^{*}(\bar{D}), \nabla_{\bar{\omega}}\right)$ where $\bar{D}$ is the divisor defined by the homogeneous part $\bar{P}_{j}$ of $P_{j}$ and $\bar{\omega}=\sum \alpha_{j} d \bar{P}_{j} / \bar{P}_{j}$. Using acyclicity of homogeneous case and the standard argument of filtered complex, in $\S 5$, we shall prove the vanishing theorem for twisted rational de Rham cohomology under a certain regularity conditions. In case each $P_{j}$ is linear and the arrangement $\left\{P_{j}=0\right\}_{1 \leq j \leq m}$ is

Received July 15, 1993. 
in general position, the integral $\int U d u_{1} \wedge \cdots \wedge d u_{n}$ has been studied in some special cases and the studies of P. Appell, E. Picard and G. Lauricella are classical. Since the integral is a multivalued holomorphic function on an open set of the complex Grassmann manifold $G(n+1, m+1)$ of $(n+1)$-dimensional subspaces of a complex $(m+1)$-dimensional vector space, we call, after the naming of [MSY], the integral the hypergeometric function of type $(n+1, m+1)$. To know the basic properties of the hypergeometric function, in the last section we shall study the degree filtration on $\Omega^{\circ}(\log D)$ and the structure of the associated graded complex in great detail, which leads to determination of the structure of $H^{n}\left(\Omega^{\circ}(\log D), \nabla_{\omega}\right)$. By using the structure of $G r_{\mu}^{F} \Omega^{\cdot}(\log D)$, we can unify the study [Ao] of twisted rational de Rham cohomology in irregular singular case and the recent study [O-T] of arrangements and Milnor fibers. This unification enables us to determine the structure of the cohomology in many interesting cases, which will be described in our forthcoming paper [AKOT].

Acknowledgement. The author would like to express his sincere gratitude to Professors J. Kaneko and T. Yamazaki who communicated their results to him in their private letters.

\section{\$1. Preliminaries on twisted rational de Rham complexes}

1.1. Let $\mathbf{C}^{n}$ be a complex $n$-dimensional affine space with coordinates $u=$ $\left(u_{1}, \ldots, u_{n}\right)$ and let $P_{j}(u), 1 \leq j \leq m$, be non-constant polynomials in $u_{1}, \ldots, u_{n}$. We set

$\Omega^{p}\left(\mathbf{C}^{n}\right):$ space of polynomial $p$-forms,

$U(u):=\prod_{j=1}^{m} P_{j}(u)^{\alpha_{j}}$,

$D$ : the divisor defined by $P:=P_{1} \cdots P_{m}$,

$X:=\mathbf{C}^{n}-D:$ a complex affine manifold,

$\omega:=d U / U=\sum_{j=1}^{m} \alpha_{j} \frac{d P_{j}}{P_{j}}:$ an integrable holomorphic 1-form on $X$,

$d$ : the exterior differentiation on $\mathbf{C}^{n}$,

$\nabla_{\omega}:=d+\omega \wedge$ : covariant differentiation with respect to $\omega$,

$\mathscr{L}_{\omega}$ : complex local system on $X$ of solutions of $\nabla_{\omega} h=0$,

$\Omega^{p}(* D)$ : space of rational $p$-forms with poles along $D$.

As is shown in [R-T], in the case of arrangement of hyperplanes which is not normal crossing, the exterior algebra generated by $d P_{j} / P_{j}, 1 \leq j \leq m$, over the 
affine coordinate ring of $X$ is smaller than the complex of logarithmic forms in the sense of K. Saito. Since this gap is essential in our later application in $\S 6$, we begin by reviewing the definition of logarithmic forms in the sense of Saito:

Definition ([S1]). A rational $p$-form $\varphi \in \Omega^{p}(* D)$ is called a logarithmic $p$-form along $D$ if $P \varphi$ and $d P \wedge \varphi$ are polynomial forms. We denote by $\Omega^{p}(\log D)$ the space of logarithmic $p$-forms along $D$.

1.2. Simple computations show that $\Omega^{*}(* D)$ is closed under the exterior differentiation and exterior products with $\frac{d P_{j}}{P_{j}}, 1 \leq j \leq m$, and hence it is also closed under the covariant differentiation $\nabla_{\omega}$. Therefore $\left(\Omega^{*}(* D), \nabla_{\omega}\right)$ is a complex whose cohomology is denoted by $H^{p}\left(\Omega^{*}(* D), \nabla_{\omega}\right)$ for $p=0,1, \ldots$ The Grothendiek-Deligue comparison theorem asserts, in our case, that there exists a canonical isomorphism

$$
H^{p}\left(X, \mathscr{L}_{\omega}\right) \simeq H^{p}\left(\Omega^{\cdot}(* D), \nabla_{\omega}\right)
$$

for $p=0,1, \ldots$, where $H^{p}\left(X, \mathscr{L}_{\omega}\right)$ is cohomology of $X$ with coefficients in the local system $\mathscr{L}_{\omega}$.

By the definition of logarithmic forms and simple computations, we can see easily that $\Omega^{*}(\log D)$ is closed under the exterior differentiation and the exterior product with $\frac{d P_{j}}{P_{j}}, 1 \leq j \leq m$, and hence it is closed under the covariant differentiation $\nabla_{\omega}$. Therefore the logarithmic complex $\left(\Omega^{\circ}(\log D), \nabla_{\omega}\right)$ is a subcomplex of the twisted rational de Rham complex $\left(\Omega^{*}(* D), \nabla_{\omega}\right)$; the cohomology of the logarithmic complex is written as $H^{p}\left(\Omega^{*}(\log D), \nabla_{\omega}\right)$. We shall study in $\S \S 5$ and 6 when the natural inclusion $\left(\Omega^{\circ}(\log D), \nabla_{\omega}\right) \rightarrow\left(\Omega^{*}(* D), \nabla_{\omega}\right)$ induces an isomorphism of the cohomology of these complexes.

1.3. For the later application in $\S 6$, we prove a property of logarithmic forms.

Lemma 1.3.1. We suppose that the polynomials $P_{i}$ and $P_{j}$ are relatively prime for $i \neq j$ and that $\alpha_{j} \neq 1$ for $1 \leq j \leq m$; then $\varphi \in \Omega^{p}(* D)$ is logarithmic along $D$ if and only if

$$
\varphi \in \frac{1}{P} \Omega^{p}\left(\mathbf{C}^{n}\right) \text { and } \nabla_{\omega} \varphi \in \frac{1}{P} \Omega^{p+1}\left(\mathbf{C}^{n}\right)
$$


Proof. We set $\varphi=\beta / P, \beta \in \Omega^{p}\left(\mathbf{C}^{n}\right)$; then we have

$$
\nabla_{\omega} \varphi=\frac{1}{P}\left\{d \beta+\sum_{j=1}^{m}\left(\alpha_{j}-1\right) \frac{d P_{j}}{P_{j}} \wedge \beta\right\}
$$

and hence $\sum_{j=1}^{m}\left(\alpha_{j}-1\right) \frac{d P_{j}}{P_{j}} \wedge \beta \in \Omega^{p+1}\left(\mathbf{C}^{n}\right)$. Since $P_{j}$ 's are relatively prime, each summand $\left(\alpha_{j}-1\right) \frac{d P_{j}}{P_{j}} \wedge \beta$ is in $\Omega^{p+1}\left(\mathbf{C}^{n}\right)$. By assumption, $\alpha_{j} \neq 1$ and hence $\frac{d P_{j}}{P_{j}} \wedge \beta \in \Omega^{p+1}\left(\mathbf{C}^{n}\right)$, which means that $\varphi$ is logarithmic along $D$.

\section{§2. The de Rham-Saito division lemma and a representation of logarithmic forms}

For a proof of vanishing of twisted de Rham cohomology, we will explicitly write down logarithmic $p$-forms along $D$ as a sum of exterior products of $\frac{d P_{j}}{P_{j}}$ $(1 \leq j \leq m)$ and polynomial forms. To the end we shall prepare some algebraic tools whose explanations are found in standard textbooks on commutative algebras.

2.1. Let $A$ be a noetherian commutative ring and let $a$ be an ideal of $A$. A sequence of elements $f_{1}, \ldots, f_{t}$ in $\mathfrak{a}$ is said to be regular if $f_{1}$ is a non-zero-divisor of $A$ and $f_{i}$ is a non-zero-divisor of $A /\left(f_{1}, \ldots, f_{i-1}\right)$ for $2 \leq i \leq t$. Then the a-depth of $A$ is defined as the maximal length $q$ of regular sequence $f_{1}, \ldots, f_{q}$ in a and denoted by $\operatorname{depth}_{\mathfrak{a}} A$.

Let $M$ be a free $A$-module of rank $n$ whose free basis is $e_{1}, \ldots, e_{n}$. We denote by $\Lambda^{p} M$ the $p$-th exterior power of $M$; Notice that $\Lambda^{0} M=A$ and $\Lambda^{-1} M=0$. Let $\omega_{1}, \ldots, \omega_{r}$ be elements of $M$; then $\omega_{1} \wedge \cdots \wedge \omega_{r}$ can be written as

$$
\omega_{1} \wedge \cdots \wedge \omega_{r}=\sum_{1 \leq i_{1}<\cdots<i_{r} \leq n} a_{i_{1} \cdots i_{r}} e_{i_{1}} \wedge \cdots \wedge e_{i_{r}}, \quad a_{i_{1} \cdots i_{r}} \in A .
$$

We denote by $a$ the ideal of $A$ generated by the coefficients $a_{i_{1} \ldots i_{r}}, 1 \leq i_{1}<\cdots$ $<i_{r} \leq n$ and set

$$
\begin{aligned}
& Z^{p}:=\left\{\varphi \in \Lambda^{p} M \mid \omega_{1} \wedge \cdots \wedge \omega_{r} \wedge \varphi=0\right\} \\
& H^{p}:=Z^{p} / \sum_{k=1}^{r} \omega_{k} \wedge \Lambda^{p-1} M
\end{aligned}
$$

then we have 
Lemma 2.1.1. (The de Rham-Saito division lemma, [deR], [S2]).

(1) There exists an integer $\nu \geq 0$ such that $\mathrm{a}^{\nu} H^{p}=0$ for $p=0, \ldots, n$.

(2) $H^{p}=0$ for $0 \leq p<\operatorname{depth}_{\mathrm{a}} A$.

2.2. Let $\bar{P}_{j}(u)$ be non-constant homogeneous polynomials in $u_{1}, \ldots, u_{n}$ and by abusing notations we denote by $\left(d \bar{P}_{j_{1}} \wedge \cdots \wedge d \bar{P}_{j_{r}}, \bar{P}_{j_{1}}, \ldots, \bar{P}_{j_{r}}\right)$ the ideal of polynomial ring $\mathbf{C}\left[u_{1}, \ldots, u_{n}\right]$ generated by $\bar{P}_{j_{1}}, \ldots, \bar{P}_{j_{r}}$ and the minors $\frac{\partial\left(\bar{P}_{j_{1}}, \ldots, \bar{P}_{j_{r}}\right)}{\partial\left(u_{j_{1}}, \ldots, u_{j_{r}}\right)}, 1 \leq i_{1}<\cdots<i_{r} \leq n$, of the Jacobian matrix $\left(\frac{\partial P_{j_{k}}}{\partial u_{i}}\right), 1 \leq k$ $\leq r, 1 \leq i \leq n$. Notice that the minors are the coefficients of $d \bar{P}_{j_{1}} \wedge \cdots \wedge d \bar{P}_{j_{r}}$ with respect to the basis $d u_{i_{1}} \wedge \cdots \wedge d u_{i_{r}}, 1 \leq i_{1}<\cdots<i_{r} \leq n$ :

$$
d \bar{P}_{j_{1}} \wedge \cdots \wedge d \bar{P}_{j_{r}}=\sum_{i_{1}<\cdots<i_{r}} \frac{\partial\left(\bar{P}_{j_{1}}, \ldots, \bar{P}_{j_{r}}\right)}{\partial\left(u_{i_{1}}, \ldots, u_{i_{r}}\right)} d u_{i_{1}} \wedge \cdots \wedge d u_{i_{r}} .
$$

We make the following assumption:

Assumption 2.2.1. (1) For $1 \leq r \leq \min \{m, n-1\}$, the algebraic set defined by the ideal $\left(d \bar{P}_{j_{1}} \wedge \cdots \wedge d \bar{P}_{j_{r}}, \bar{P}_{j_{1}}, \ldots, \bar{P}_{j_{r}}\right)$ is either empty or of dimension 0 . In algebraic words,

(2) $\bar{P}_{j_{1}}, \ldots, \bar{P}_{j_{s}}$ form a regular sequence in $\mathbf{C}\left[u_{1}, \ldots, u_{n}\right]$ for $1 \leq s \leq \min \{m, n\}$.

Under our assumption, we obtain the following Lemma, which is essential in the proof of Proposition 2.2.3 below.

Lemma 2.2.2. We suppose Assumption 2.2.1. Let $r+p \leq n-1$ and let $\phi$ be a polynomial p-form such that

$$
d \bar{P}_{j_{1}} \wedge \cdots \wedge d \bar{P}_{j_{r}} \wedge \phi \equiv 0 \quad\left(\bmod \bar{P}_{j_{1}}, \ldots, \bar{P}_{j_{r}}\right)
$$

then

$$
\phi \equiv 0 \quad\left(\bmod d \bar{P}_{j_{1}}, \ldots, d \bar{P}_{j_{r}}, \bar{P}_{j_{1}}, \ldots, \bar{P}_{j_{r}}\right)
$$

Proof. We set

$$
\begin{aligned}
& B:=\mathbf{C}\left[u_{1}, \ldots, u_{n}\right], \\
& I:=\left(d \bar{P}_{j_{1}} \wedge \cdots \wedge d \bar{P}_{j_{r}}, \bar{P}_{j_{1}}, \ldots, \bar{P}_{j_{r}}\right), \\
& A:=B /\left(\bar{P}_{j_{1}}, \ldots, \bar{P}_{j_{r}}\right), \\
& a:=I /\left(\bar{P}_{j_{1}}, \ldots, \bar{P}_{j_{r}}\right) ; \text { an ideal of } A,
\end{aligned}
$$


$\pi: B \rightarrow A$; the canonical homomorphism.

Then we have $I=\pi^{-1}(\mathfrak{a})$ and hence $B / I \simeq A / \mathfrak{a}$. By virtue of Lemma 2.1.1, it suffices to show that either $\mathfrak{a}=A$ or $\operatorname{depth}_{\mathfrak{a}} A=n-r$. We divide into two cases. In case $I=B$, we have $a=A$ and hence part (i) of Lemma 2.1.1 gives $H^{p}=0$ for $p=0, \ldots, n$. In case $I \neq B$, by Assumption we get height $I=n$. On the other hand, it is known (see [M], p. 105) that

$$
\operatorname{depth}_{\mathrm{a}} A=\inf \left\{\operatorname{depth} A_{\mathrm{m}} \mid \mathrm{m} \in V(\mathfrak{a})\right\}
$$

where $V(\mathfrak{a})$ is the set of prime ideals $\mathrm{m}$ such that $\mathfrak{a} \subset \mathrm{m}$ and $A_{\mathrm{m}}$ is the localization of $A$ at a prime ideal $\mathrm{m}$. Since height $I=n$, we have $\operatorname{dim} A / \mathfrak{a}=\operatorname{dim} B / I=$ 0 and hence $\mathfrak{m}$ is a maximal ideal of $A$. We set $\mathfrak{n}:=\pi^{-1}(\mathfrak{m})$; then $\mathfrak{n}$ is a maximal ideal of $B$ and we have that $B_{\mathfrak{n}}$ is an $n$-dimensional regular local ring and

$$
A_{\mathrm{m}} \simeq B_{\mathrm{n}} /\left(\bar{P}_{j_{1}}, \ldots, \bar{P}_{j_{r}}\right) B_{\mathrm{n}} .
$$

Since by assumption $\bar{P}_{j_{1}}, \ldots, \bar{P}_{j_{r}}$ is a $B$-regular sequence, it is also a $B_{\mathfrak{n}}$-regular sequence ane hence we can be conclude that $A_{\mathrm{m}}$ is a Cohen-Macaulay local ring. This implies that

$$
\begin{aligned}
\operatorname{depth} A_{\mathrm{m}} & =\operatorname{dim} A_{\mathrm{m}} \\
& =\operatorname{dim} B_{\mathfrak{r}}-r \quad(\text { by }(2.2 .2) \text { and }[\mathrm{M}], \text { p. 105) } \\
& =n-r,
\end{aligned}
$$

and hence by (2.2.1), we get $\operatorname{depth}_{\mathfrak{a}} A=n-r$.

Remark. Our first proof ([K-N]) was rather complicated; the proof here is due to T. Yamazaki, who kindly communicated it to the author ([Ya]).

Proposition 2.2.3 (Representation theorem for logarithmic forms). Let $\bar{P}_{j}(u)$, $1 \leq j \leq m$, be polynomials satisfying Assumption 2.2.1. Let $0 \leq p \leq n-2$ be an integer and let $\phi$ be a polynomial p-form such that

$$
d \bar{P}_{j} \wedge \phi \equiv 0\left(\bmod \bar{P}_{j}\right) \quad \text { for } \quad 1 \leq j \leq m .
$$

Then $\phi$ can be written in the form

$$
\begin{aligned}
\phi=\bar{P}_{1} \cdots \bar{P}_{m}\left\{\phi_{0}+\sum_{j=1}^{m} \frac{d \bar{P}_{j}}{\bar{P}_{j}} \wedge \phi_{j}+\cdots\right. \\
\left.\cdots+\sum_{1 \leq j_{1}<\cdots<j_{p} \leq m} \frac{d \bar{P}_{j_{1}}}{\bar{P}_{j_{1}}} \wedge \cdots \wedge \frac{d \bar{P}_{j_{p}}}{\bar{P}_{j_{p}}} \phi_{j_{1} \cdots j_{p}}\right\}
\end{aligned}
$$


where $\phi_{j_{1} \cdots j_{\nu}}$ is a polynomial $(p-\nu)$-form for $1 \leq j_{1}<\cdots<j_{\nu} \leq m$ and $\nu=0, \ldots, p$.

Proof. We shall prove Proposition 2.2 .3 by induction on the number $m$ of polynomials $\bar{P}_{j}, 1 \leq j \leq m$. In case $m=1$, by (2.2.3), $0 \leq p \leq n-2$ and Lemma 2.2.2, we have

$$
\phi \equiv 0\left(\bmod d \bar{P}_{1}, \bar{P}_{1}\right)
$$

Hence $\phi$ can be written as

$$
\phi=\bar{P}_{1} \phi_{0}+d \bar{P}_{1} \wedge \phi_{1}=\bar{P}_{1}\left\{\phi_{0}+\frac{d \bar{P}_{1}}{\bar{P}_{1}} \wedge \phi_{1}\right\}
$$

where $\phi_{0} \in \Omega^{p}\left(\mathbf{C}^{n}\right)$ and $\phi_{1} \in \Omega^{p-1}\left(\mathbf{C}^{n}\right)$, which shows that Proposition 2.2.3 holds for $m=1$. We assume Proposition 2.2 .3 is true for $m$; let $\phi \in \Omega^{p}\left(\mathbf{C}^{n}\right)$ such that $d \bar{P}_{j} \wedge \psi \equiv 0\left(\bmod \bar{P}_{j}\right)$ for $1 \leq j \leq m+1$. By induction, $\phi$ can be written in the form (2.2.4). Let $N$ be the largest integer for which there exists some $\phi_{j_{1} \ldots j_{N}} \neq 0$ in (2.2.4). By induction on $N$, we shall show that $\phi$ can be written in the form

$$
\begin{gathered}
\psi=\bar{P}_{1} \cdots \bar{P}_{m} \bar{P}_{m+1}\left\{\gamma_{0}+\sum_{j=1}^{m+1} \frac{d \bar{P}_{j}}{\bar{P}_{j}} \wedge \gamma_{1}+\cdots\right. \\
\left.\cdots+\sum_{1 \leq j_{1}<\cdots<j_{p} \leq m+1} \frac{d \bar{P}_{j_{1}}}{\bar{P}_{j_{1}}} \wedge \cdots \wedge \frac{d \bar{P}_{j_{p}}}{\bar{P}_{j_{p}}} \gamma_{j_{1} \cdots j_{p}}\right\} .
\end{gathered}
$$

In case $N=0$, we have $\phi=\bar{P}_{1} \cdots \bar{P}_{m} \psi_{0}$. Since $d \bar{P}_{m+1} \wedge \phi \equiv 0\left(\bmod \bar{P}_{m+1}\right)$, we get

$$
\bar{P}_{1} \cdots \bar{P}_{m} d \bar{P}_{m+1} \wedge \phi_{0} \equiv 0 \quad\left(\bmod \bar{P}_{m+1}\right) .
$$

Since $0 \leq p \leq n-2$, Assumption 2.2.1, (2) implies that $\left\{P_{j} ; P_{m+1}\right\}$ form a regular sequence for $1 \leq j \leq m$ and hence Lemma 2.2 .2 yields $\phi_{0} \equiv 0\left(\bmod d \bar{P}_{m+1}\right.$, $\left.\bar{P}_{m+1}\right)$. Thus $\phi_{0}$ can be written in the form

$$
\phi_{0}=\bar{P}_{m+1} \alpha+d \bar{P}_{m+1} \wedge \beta, \alpha \in \Omega^{p}\left(\mathbf{C}^{n}\right), \beta \in \Omega^{p-1}\left(\mathbf{C}^{n}\right)
$$

and hence

$$
\phi=\bar{P}_{1} \cdots \bar{P}_{m+1}\left\{\alpha+\frac{d \bar{P}_{m+1}}{\bar{P}_{m+1}} \wedge \beta\right\},
$$

which means that Proposition 2.2.3 holds for $N=0$. Suppose the statement is true for $N-1$ and we shall show that it holds for $N$. For simplicity we focus our 
attention on the index $(1, \ldots, N)$; by definition of $N, \phi_{j_{1} \ldots j_{\nu}}=0$ for $\nu \geq N+1$ and hence (2.2.4) yields

$$
\phi-\bar{P}_{N+1} \cdots \bar{P}_{m} d \bar{P}_{1} \wedge \cdots \wedge d \bar{P}_{N} \wedge \phi_{1 \cdots N} \equiv 0\left(\bmod \bar{P}_{1} \cdots \bar{P}_{N}\right) .
$$

Since $N \leq p \leq n-2$, Assumption 2.2.1, (2) implies that $\left\{\bar{P}_{1}, \ldots, \bar{P}_{N}, \bar{P}_{j}, \bar{P}_{m+1}\right\}$ form a regular sequence for $N+1 \leq j \leq m$. Using $d \bar{P}_{m+1} \wedge \phi \equiv 0\left(\bmod \bar{P}_{m+1}\right)$, we have

$$
d \bar{P}_{1} \wedge \cdots \wedge d \bar{P}_{N} \wedge d \bar{P}_{m+1} \wedge \phi_{1 \ldots N} \equiv 0\left(\bmod \bar{P}_{1}, \ldots, \bar{P}_{N}, \bar{P}_{m+1}\right) .
$$

Since $\phi_{1 \cdots N} \in \Omega^{p-N}\left(\mathbf{C}^{n}\right)$ and $N \leq p \leq n-2$, Lemma 2.2 .2 yields

$$
\phi_{1 \ldots N} \equiv 0\left(\bmod d \bar{P}_{1}, \ldots, d \bar{P}_{N}, d \bar{P}_{m+1}, \bar{P}_{1}, \ldots, \bar{P}_{N}, \bar{P}_{m+1}\right)
$$

and hence we can write $\phi_{1 \ldots N}$ as

$$
\begin{gathered}
\phi_{1 \cdots N}=\sum_{j=1}^{N} \bar{P}_{j} \alpha_{1 \cdots N ; j}+\bar{P}_{m+1} \alpha_{1 \cdots N ; m+1} \\
\quad+\sum_{j=1}^{N} d \bar{P}_{j} \wedge \beta_{1 \cdots N ; j}+d \bar{P}_{m+1} \wedge \beta_{1 \cdots N ; m+1}, \\
\alpha_{1 \cdots N ; j} \in \Omega^{p-N}\left(\mathbf{C}^{n}\right), \quad \beta_{1 \cdots N ; j} \in \Omega^{p-N-1}\left(\mathbf{C}^{n}\right) .
\end{gathered}
$$

By the same reasoning we have

$$
\begin{gathered}
\phi_{\rho_{1} \cdots j_{N}}=\sum_{k=1}^{N} \bar{P}_{j_{k}} \alpha_{j_{1} \cdots j_{N} ; j_{k}}+\bar{P}_{m+1} \alpha_{j_{1} \cdots j_{N} ; m+1} \\
\quad+\sum_{k=1}^{N} d \bar{P}_{j_{k}} \wedge \beta_{j_{1} \cdots j_{N} ; j_{k}}+d \bar{P}_{m+1} \wedge \beta_{j_{1} \cdots j_{N} ; m+1}, \\
\alpha_{j_{1} \ldots j_{N} ; j_{k}} \in \Omega^{p-N}\left(\mathbf{C}^{n}\right), \quad \beta_{1_{1} \cdots j_{N} ; j_{k}} \in \Omega^{p-N-1}\left(\mathbf{C}^{n}\right) .
\end{gathered}
$$

Substituting (2.2.6) into (2.2.4), we get

$$
\begin{gathered}
\varphi=\bar{P}_{1} \cdots \bar{P}_{m}\left\{\sum_{\nu=0}^{N-1} \sum_{1 \leq j_{1}<\cdots<j_{\nu} \leq m} \frac{d \bar{P}_{j_{1}}}{\bar{P}_{j_{1}}} \wedge \cdots \wedge \frac{d \bar{P}_{j_{\nu}}}{\bar{P}_{j_{\nu}}} \wedge \phi_{j_{1} \cdots j_{\nu}}\right\} \\
+\bar{P}_{1} \cdots \bar{P}_{m}\left\{\sum _ { 1 \leq j _ { 1 } < \cdots < j _ { N } \leq m } \frac { d \overline { P } _ { j _ { 1 } } } { \overline { P } _ { j _ { 1 } } } \wedge \cdots \wedge \frac { d \overline { P } _ { j _ { N } } } { \overline { P } _ { j _ { N } } } \wedge \left(\sum_{k=1}^{N} \bar{P}_{j_{k}} \alpha_{j_{1} \cdots j_{N} ; j_{k}}\right.\right. \\
\left.\left.+\bar{P}_{m+1} \alpha_{j_{1} \cdots j_{N} ; m+1}\right)\right\}
\end{gathered}
$$




$$
\begin{gathered}
+\bar{P}_{1} \cdots \bar{P}_{m}\left\{\sum _ { 1 \leq j _ { 1 } < \cdots < j _ { N } \leq m } \frac { d \overline { P } _ { j _ { 1 } } } { \overline { P } _ { j _ { 1 } } } \wedge \cdots \wedge \frac { d \overline { P } _ { j _ { N } } } { \overline { P } _ { j _ { N } } } \wedge \left(\sum_{k=1}^{N} d \bar{P}_{j_{k}} \wedge \beta_{j_{1} \cdots j_{N} ; j_{k}}\right.\right. \\
\left.\left.+d \bar{P}_{m+1} \wedge \beta_{j_{1} \cdots j_{N} ; m+1}\right)\right\} .
\end{gathered}
$$

Notice that the last term in the right hand side in (2.2.7) reduces to

$$
\bar{P}_{1} \cdots \bar{P}_{m}\left\{\sum_{1 \leq j_{1}<\cdots<j_{N} \leq m} \frac{d \bar{P}_{j_{1}}}{\bar{P}_{j_{1}}} \wedge \cdots \wedge \frac{d \bar{P}_{j_{N}}}{\bar{P}_{j_{N}}} \wedge d \bar{P}_{m+1} \wedge \beta_{j_{1} \cdots j_{N} ; m+1}\right\} .
$$

We set

$$
\begin{aligned}
\eta= & \phi-\bar{P}_{1} \cdots \bar{P}_{m+1}\left\{\sum_{1 \leq j_{1}<\cdots<j_{N} \leq m+1} \frac{d \bar{P}_{j_{1}}}{\bar{P}_{j_{1}}} \wedge \cdots \wedge \frac{d \bar{P}_{j_{N}}}{\bar{P}_{j_{N}}} \wedge \alpha_{j_{1} \cdots j_{N} ; m+1}\right. \\
& \left.+\sum_{1 \leq j_{1}<\cdots<j_{N} \leq m} \frac{d \bar{P}_{j_{1}}}{\bar{P}_{j_{1}}} \wedge \cdots \wedge \frac{d \bar{P}_{j_{N}}}{\bar{P}_{j_{N}}} \wedge \frac{d \bar{P}_{m+1}}{\bar{P}_{m+1}} \wedge \beta_{j_{1} \cdots j_{N} ; m+1}\right\}
\end{aligned}
$$

Using (2.2.7), we can write $\eta$ in the form

$$
\begin{aligned}
\eta= & \bar{P}_{1} \cdots \bar{P}_{m}\left\{\sum_{\nu=0}^{N-1} \sum_{1 \leq j_{1}<\cdots<j_{\nu} \leq m} \frac{d \bar{P}_{j_{1}}}{\bar{P}_{j_{1}}} \wedge \cdots \wedge \frac{d \bar{P}_{j_{\nu}}}{\bar{P}_{j_{\nu}}} \wedge \eta_{j_{1} \cdots j_{\nu}}\right\}, \\
& \eta_{j_{1} \cdots j_{\nu}} \in \Omega^{p-\nu}\left(\mathbf{C}^{n}\right) .
\end{aligned}
$$

By $(2.2 .8)$ and $d \bar{P}_{j} \wedge \phi \equiv 0\left(\bmod \bar{P}_{j}\right), 1 \leq j \leq m+1$, we see that $d \bar{P}_{j} \wedge \eta \equiv 0$ $\left(\bmod \bar{P}_{j}\right)$ for $1 \leq j \leq m+1$. This, together with (2.2.9), means that $\eta$ satisfies the assumption of induction and hence it is written in the form

$$
\begin{aligned}
\eta= & \bar{P}_{1} \cdots \bar{P}_{m} \bar{P}_{m+1}\left\{\tilde{\eta}_{0}+\sum_{j=1}^{m+1} \frac{d \bar{P}_{j}}{\bar{P}_{j}} \wedge \tilde{\eta}_{j}+\cdots\right. \\
& \left.+\sum_{1 \leq j_{1}<\cdots<j_{p} \leq m+1} \frac{d \bar{P}_{j_{1}}}{\bar{P}_{j_{1}}} \wedge \cdots \wedge \frac{d \bar{P}_{j_{p}}}{\bar{P}_{j_{p}}} \wedge \tilde{\eta}_{j_{1} \cdots j_{p}}\right\} .
\end{aligned}
$$

Substituting (2.2.10) into (2.2.8), we see that $\phi$ can be written in the form (2.2.5). This completes induction.

Remark. A logarithmic $p$-form $\varphi,(0 \leq p \leq n-2)$ is, by definition, written as $\varphi=\phi / \bar{P}$ where $\phi \in \Omega^{p}\left(\mathbf{C}^{n}\right)$, which satisfies $\frac{d \bar{P}_{j}}{\bar{P}_{j}} \wedge \phi \in \Omega^{p+1}\left(\mathbf{C}^{n}\right)$ for $1 \leq j$ $\leq m$. Hence $\phi$ satisfies the condition in Proposition 2.2.3. Therefore $\varphi$ is written in the form of the second factor of the right hand side of (2.2.4). Notice that $\varphi$ has not logarithmic pole along the hyperplane at infinity. 


\section{§3. Acyclicity of the twisted rational de Rham complex in homogeneous case}

3.1. Let $\bar{P}_{j}(u), 1 \leq j \leq m$, be homogeneous polynomials in $u=\left(u_{1}, \ldots, u_{n}\right)$ and let $\bar{D}_{j}$ be the divisors defined by $\bar{P}_{j}$; we set

$$
\begin{aligned}
& \bar{D}:=\sum_{j=1}^{m} \bar{D}_{j}, \quad \bar{U}(u)=\prod_{j=1}^{m} \bar{P}_{j}(u)^{\alpha_{j}}, \\
& \bar{\omega}=d \bar{U} / \bar{U}, \quad \nabla_{\bar{\omega}}=d+\bar{\omega}_{\wedge} .
\end{aligned}
$$

As is explained in 1.2 , the logarithmic complex $\left(\Omega^{\circ}(\log \bar{D}), \nabla_{\bar{\omega}}\right)$ with poles along $\bar{D}$ is a subcomplex of the twisted rational de Rham complex $\left(\Omega^{*}(* \bar{D}), \nabla_{\bar{\omega}}\right)$. To compute cohomology of these complexes, we shall introduce some operators acting on them. Let $v=\sum_{j=1}^{n} u_{i} \frac{\partial}{\partial u_{i}}$ be the Euler vector field on $\mathbf{C}^{n}$ and we set as follows:

$i_{v}$ : the interior product with respect to $v$,

$L_{v}$ : the Lie differentiation with respect to $v$,

$\bar{\omega}_{\wedge}$ : the exterior product of $\bar{\omega}$.

It is easily checked that exterior differentiation $d, i_{v}, L_{v}$ and $\bar{\omega}_{\wedge}$ act on the complexes $\Omega^{*}(* \bar{D})$ and $\Omega^{*}(\log \bar{D})$ and hence $\nabla_{\bar{\omega}}$ also acts on them. Recall that $d$ and $i_{v}$ are skew-derivations of $\Omega^{*}(* \bar{D})$ of degree +1 and -1 , respectively; $L_{v}$ is a derivation of $\Omega^{*}(* D)$ of degree 0 .

3.2. In the following we summarize the fundamental relations among the operators defined in the previous subsection:

(1) The commutation relations:

$$
\left[L_{v}, d\right]=0,\left[L_{v}, i_{v}\right]=0,\left[L_{v}, \bar{\omega}_{\wedge}\right]=0,\left[L_{v}, \nabla_{\bar{\omega}}\right]=0 ;
$$

(2) The homotopy formula of H. Cartan:

$$
d \circ i_{v}+i_{v} \circ d=L_{v}
$$

which gives rise to the homotopy formula for $\nabla_{\bar{\omega}}$ :

$$
\nabla_{\bar{\omega}} \circ i_{v}+i_{v} \circ \nabla_{\bar{\omega}}=L_{v}+\sum_{j=1}^{m} l_{j} \alpha_{j}
$$

where $\bar{\omega}=\sum_{j=1}^{m} \alpha_{j} \frac{d \bar{P}_{j}}{\bar{P}_{j}}$ and we have set $l_{j}=\operatorname{deg} \bar{P}_{j}$. 
3.3. Let $g(u)$ be a homogeneous polynomial of degree $\mu$. Since $L_{v}(g)=\mu g$ and $L_{v}\left(d u_{i}\right)=d u_{i}$, we have

$$
L_{v}\left(g(u) d u_{i_{1}} \wedge \cdots \wedge d u_{i_{p}}\right)=(\mu+p) g(u) d u_{i_{1}} \wedge \cdots \wedge d u_{i_{p}}
$$

Thus a polynomial $p$-form $\phi \in \Omega^{p}\left(\mathbf{C}^{n}\right)$ is said to be homogeneous of degree $\mu$ if $L_{v}(\phi)=\mu \phi$ holds. Notice that when $\phi$ has the form

$$
\phi=\sum_{1 \leq i_{1}<\cdots<i_{p} \leq n} \phi_{i_{1} \cdots i_{p}}(u) d u_{i_{1}} \wedge \cdots \wedge d u_{i_{p}},
$$

$\phi$ is homogeneous of degree $\mu$ if and only if each $\phi_{i_{1} \cdots i_{p}}(u)$ is a homogeneous polynomial of degree $\mu-p$. We shall extend the definition of degree to rational $p$-forms $\Omega^{p}(* D)$ with poles along $\bar{D}$. First notice that $L_{v}(1 / \bar{P})=-l / \bar{P}$ for $\operatorname{deg} \bar{P}=l$. A rational $p$-form $\varphi \in \Omega^{p}(* \bar{D})$ can be written as $\varphi=\beta / \bar{P}^{k}$ for some positive integer $k$ and some polynomial $p$-form $\beta$; then $\varphi$ is said to be homogeneous of degree $\mu(\mu \in \mathbf{Z})$ if $\beta$ is of homogeneous of degree $\mu+k l$. It is easily checked that this definition is independent of the choice of expression of $\varphi$ as $\beta / \bar{P}^{k}$ and it is characterized by the relation $L_{v}(\varphi)=\mu \varphi$. We denote by $\Omega^{p}(* \bar{D})_{\mu}$ the subspace of $\Omega^{p}(* \bar{D})$ consisting all homogeneous $p$-forms of degree $\mu$, which is the eigenspace of $L_{v}$ belonging to the eigenvalue $\mu . \Omega^{*}(* D)$ is decomposed into the direct sum of eigenspaces of $L_{v}$ :

$$
\Omega^{p}(* \bar{D})=\bigoplus_{\mu \in \mathbf{Z}} \Omega^{p}(* \bar{D})_{\mu} .
$$

Since $L_{v}$ acts on the subspace $\Omega^{p}(\log \bar{D})$ of $\Omega^{p}(* \bar{D})$, by setting $\Omega^{p}(\log \bar{D})_{\mu}:=$ $\Omega^{p}(\log \bar{D}) \cap \Omega^{p}(* \bar{D})_{\mu}$, we have

$$
\Omega^{p}(\log \bar{D})=\bigoplus_{\mu \in \mathbf{Z}} \Omega^{p}(\log \bar{D})_{\mu} .
$$

3.4. By the commutation relation (3.2.1), the operators $d, i_{v}, \bar{\omega}_{\wedge}$ and $\nabla_{\bar{\omega}}$ act on eigenspaces $\Omega^{*}(* \bar{D})_{\mu}$ and $\Omega^{*}(\log \bar{D})_{\mu}$ and hence $\left(\Omega^{*}(* \bar{D})_{\mu}, \nabla_{\bar{\omega}}\right)$ and $\left(\Omega^{*}(* \bar{D})_{\mu}, \nabla_{\bar{\omega}}\right)$ are subcomplexes of $\left(\Omega^{*}(* \bar{D}), \nabla_{\bar{\omega}}\right)$ and $\left(\Omega^{*}(\log \bar{D}), \nabla_{\bar{\omega}}\right)$, respectively. Summiag up, we have

$$
\begin{aligned}
& \left(\Omega^{*}(* \bar{D}), \nabla_{\bar{\omega}}\right)=\bigoplus_{\mu \in \mathbf{Z}}\left(\Omega^{*}(* \bar{D})_{\mu}, \nabla_{\bar{\omega}}\right), \\
& \left(\Omega^{*}(\log \bar{D}), \nabla_{\bar{\omega}}\right)=\bigoplus_{\mu \in \mathbf{Z}}\left(\Omega^{*}(\log \bar{D})_{\mu}, \nabla_{\bar{\omega}}\right) .
\end{aligned}
$$

Notice that the homotopy formula (3.2.2) for $\nabla_{\bar{\omega}}$ holds for these subcomplexes. 
Since $\left[L_{v}, \nabla_{\bar{\omega}}\right]=0$, the above decompositions induce the following ones on a cohomology level:

$$
\begin{aligned}
H^{p}\left(\Omega^{*}(* \bar{D}), \nabla_{\bar{\omega}}\right) & =\bigoplus_{\mu \in \mathbf{Z}} H^{p}\left(\Omega^{\cdot}(* \bar{D})_{\mu}, \nabla_{\bar{\omega}}\right), \\
H^{p}\left(\Omega^{\cdot}(\log \bar{D}), \nabla_{\bar{\omega}}\right) & =\bigoplus_{\mu \in \mathbf{Z}} H^{p}\left(\Omega^{\cdot}(\log \bar{D})_{\mu}, \nabla_{\bar{\omega}}\right) .
\end{aligned}
$$

Using the above decompositions and (3.2.2), we obtain

THEOREM 3.4.1. (1) If $\sum_{j=1}^{m} l_{j} \alpha_{j} \notin \mathbf{Z}$, then $H^{p}\left(\Omega^{\cdot}(* \bar{D})_{\mu}, \nabla_{\bar{\omega}}\right)=0$ for all $p$ and hence $H^{p}\left(\Omega^{*}(* \bar{D}), \nabla_{\bar{\omega}}\right)=0$ for all $p$.

(2) If $\sum_{j=1}^{m} l_{j} \alpha \neq l, l-1, l-2, \ldots$, then $H^{p}\left(\Omega^{\cdot}(\log \bar{D})_{\mu}, \nabla_{\bar{\omega}}\right)=0$ for all $p$ and hence $H^{p}\left(\Omega^{\cdot}(\log \bar{D}), \nabla_{\bar{\omega}}\right)=0$ for all $p$.

Proof. Let $\varphi$ be in $\Omega^{p}(* \bar{D})_{\mu}$ such that $\nabla_{\bar{\omega}} \varphi=0$; then by (3.2.2) and $L_{v}(\varphi)=\mu \varphi$, we get

$$
\nabla_{\bar{\omega}} \circ i_{v}(\varphi)=\left(\mu+\sum_{j=1}^{m} l_{j} \alpha_{j}\right) \varphi
$$

Since $\sum l_{j} \alpha_{j} \notin \mathbf{Z}$, we have $\mu+\sum l_{j} \alpha_{j} \neq 0$ and hence

$$
\varphi=\nabla_{\bar{\omega}}\left(\frac{i_{v}(\varphi)}{\mu+\sum l_{j} \alpha_{j}}\right)
$$

which shows $H^{p}\left(\Omega^{*}(* \bar{D})_{\mu}, \nabla_{\bar{\omega}}\right)=0$. Similarly the second statement is proved.

\section{\$4. Filtration on a logarithmic complex $\left(\Omega^{\circ}(\log D), \nabla_{\omega}\right)$}

Let $P_{j}(u), 1 \leq j \leq m$, be non-zero polynomials in $u=\left(u_{1}, \ldots, u_{n}\right)$ of which the homogeneous part of maximal degree is denoted by $\bar{P}_{j}(u)$ and we set $l_{j}:=$ $\operatorname{deg} \bar{P}_{j}$ for $1 \leq j \leq m$. We will keep using the notations listed in 1.1 and 3.1.

4.1. We define an increasing filtration on the logarithmic complex $\Omega^{\bullet}(\log D)$. To the end, let $\varphi$ be in $\Omega^{p}(\log D)$; then by definition, $\varphi$ is written as $\varphi=\alpha / P$ where $\alpha \in \Omega^{p}\left(\mathbf{C}^{n}\right)$. We may formally consider the degree of $1 / P$ as $-l:=-\sum_{j=1}^{m} l_{j}$ and hence we say that $\varphi$ is of degree $\mu$ if $\operatorname{deg} \alpha=\mu+l$.

Definition. Let $F_{\mu} \Omega^{p}(\log D)$ be the space of logarithmic $p$-forms $\varphi$ with poles along $D$ such that $\operatorname{deg} \varphi \leq \mu$. Then the family of subspaces $F_{\mu} \Omega^{p}(\log D)$, 
$\mu \geq-l+p$, defines an increasing filtration $F$ on $\Omega^{p}(\log D)$.

To show that the filtration $F$. is compatible with the covariant differentiation $\nabla_{\omega}$, we first observe that $F$. is compatible with the exterior differentiation $d$. In fact, let $\varphi=\alpha / P \in F_{\mu} \Omega^{p}(\log D)$; then $\alpha \in \Omega^{p}\left(\mathbf{C}^{n}\right), \operatorname{deg} \alpha \leq \mu+l$ and $\frac{d P}{P} \wedge$ $\alpha=: \beta \in \Omega^{p+1}\left(\mathbf{C}^{n}\right)$. Since $d \varphi=(d \alpha-\beta) / P$ and $d \alpha-\beta \in \Omega^{p+1}\left(\mathbf{C}^{n}\right)$ of degree $\leq \mu+l$, we have $d \varphi \in F_{\mu} \Omega^{p+1}(\log D)$. Since $\omega=\sum_{j=1}^{m} \alpha_{j} \frac{d P_{j}}{P_{j}}$ and $\frac{d P_{j}}{P_{j}}$ is a logarithmic 1 -form of degree 0 , we get $\omega \wedge \varphi \in F_{\mu} \Omega^{p+1}(\log D)$. Hence we obtain

$$
\nabla_{\omega} F_{\mu} \Omega^{p}(\log D) \subset F_{\mu} \Omega^{p+1}(\log D),
$$

which means that $F$. and $\nabla_{\omega}$ are compatible. This shows that $\left(F_{\mu} \Omega^{\circ}(\log D), \nabla_{\omega}\right)$, $\mu \geq-l$, are a family of subcomplexes of the logarithmic complex $\left(\Omega^{\circ}(\log D)\right.$, $\nabla_{\omega}$ ) which defines a filtration on $\Omega^{*}(\log D)$. Let

$$
G r_{\mu}^{F} \Omega^{\cdot}(\log D):=F_{\mu} \Omega^{\cdot}(\log D) / F_{\mu-1} \Omega^{\bullet}(\log D)
$$

be the associated graded complex to the filtered complex $\left(F . \Omega^{\circ}(\log D)\right.$, $\nabla_{\omega}$ ) equipped with the differential $G r_{\mu}^{F}\left(\nabla_{\omega}\right)$ induced by $\nabla_{\omega}$.

4.2. We shall compare the associated graded complex $\left(G r_{\mu}^{F} \Omega^{\circ}(\log D)\right.$, $\left.G r_{\mu}^{F}\left(\nabla_{\omega}\right)\right)$ with the subcomplex $\left(\Omega^{*}(\log \bar{D})_{\mu}, \nabla_{\bar{\omega}}\right)$ of $\left(\Omega^{\cdot}(\log \bar{D}), \nabla_{\bar{\omega}}\right)$ consisting of homogeneous logarithmic forms of degree $\mu$ introduced in 3.3. To the end we define the canonical linear mapping

$$
\sigma_{\mu}^{p}: G r_{\mu}^{F} \Omega^{p}(\log D) \rightarrow \Omega^{p}(\log \bar{D})_{\mu}
$$

as follows: let $\varphi \in F_{\mu} \Omega^{p}(\log D)$; then $\varphi=\alpha / P, \alpha \in \Omega^{p}\left(\mathbf{C}^{n}\right)$ and $\alpha \leq \mu+l$. We denote by $\bar{\alpha}$ the homogeneous part of $\alpha$ of degree $\mu+l$ and set $\bar{\varphi}:=\bar{\alpha} / \bar{P}$; then we see easily $\bar{\varphi} \in \Omega^{p}(\log \bar{D})_{\mu}$. We define the mapping $\sigma_{\mu}^{p}$ as sending $\varphi \bmod$ $F_{\mu-1} \Omega^{p}(\log D)$ to $\bar{\varphi}$; then by the definition of $\bar{\varphi}$, it is clear that $\sigma_{\mu}^{p}$ is well-defined and injective. Moreover $\sigma_{\mu}^{p \text { s }}$ s induce a morphism of complexes:

$$
\sigma_{\mu}^{\cdot}:\left(G r_{\mu}^{F} \Omega^{\cdot}(\log D), G r_{\mu}^{F}\left(\nabla_{\omega}\right)\right) \rightarrow\left(\Omega^{\cdot}(\log \bar{D})_{\mu}, \nabla_{\bar{\omega}}\right) .
$$

For let $\varphi \in F_{\mu} \Omega^{p}(\log D)$ and set $[\varphi]:=\varphi \bmod F_{\mu-1} \Omega^{p}(\log D)$ and $\bar{\varphi}:=$ $\sigma_{\mu}^{p}([\varphi]) ;$ then

$$
\nabla_{\bar{\omega}}{ }^{\circ} \sigma_{\mu}^{p}([\varphi])=d \bar{\varphi}+\sum \alpha_{j} \frac{d \bar{P}_{\jmath}}{\bar{P}_{j}} \wedge \bar{\varphi}
$$




$$
\begin{aligned}
& =\overline{\left(d \varphi+\sum \alpha_{j} \frac{d \bar{P}_{j}}{\bar{P}_{j}} \wedge \varphi\right)} \\
& =\sigma_{\mu}^{p+1} \circ G r_{\mu}^{F}\left(\nabla_{\omega}\right)([\varphi]) .
\end{aligned}
$$

4.3. We set

$$
N^{p}(\log D)_{\mu}:=\Omega^{p}(\log \bar{D})_{\mu} / \operatorname{Im} \sigma_{\mu}^{p} .
$$

Since $\sigma_{\mu}^{p}$ is injective, we have a short exact sequence of complexes:

$$
0 \rightarrow G_{\mu}^{F} \Omega^{\cdot}(\log D) \rightarrow \Omega^{*}(\log \bar{D})_{\mu} \rightarrow N^{*}(\log D)_{\mu} \rightarrow 0 .
$$

For the complex $N^{*}(\log D)_{\mu}$, we have the following

Lemma 4.3.1. If Assumption 2.2.1. holds for the homogeneous parts $\bar{P}_{j}$ of $P_{j}$, $1 \leq j \leq m$, then $\sigma_{\mu}^{p}$ is an isomorphism for $p \neq n-1$ and hence $N^{p}(\log D)_{\mu}=0$ for $p \neq n-1$.

Proof. It suffices to show $\sigma_{\mu}^{p}$ is surjective for $p \neq n-1$. Let $\bar{\varphi} \in$ $\Omega^{p}(\log \bar{D})_{\mu}, 0 \leq p \leq n-2$; then we have that $\bar{\varphi}=\bar{\alpha} / \bar{P}$ where $\bar{\alpha} \in \Omega^{p}\left(\mathbf{C}^{n}\right)$ is homogeneous of degree $\mu+l$ and $\frac{d \bar{P}_{j}}{\bar{P}_{j}} \wedge \bar{\alpha} \in \Omega^{p+1}\left(\mathbf{C}^{n}\right)$ for $1 \leq j \leq m$. By Proposition 2.2.3, we can write $\bar{\alpha}$ as follows:

$$
\begin{gathered}
\bar{\alpha}=\bar{P}_{1} \cdots \bar{P}_{m}\left\{\alpha_{0}+\sum_{j=1}^{m} \frac{d \bar{P}_{j}}{\bar{P}_{j}} \wedge \bar{\alpha}_{j}+\cdots\right. \\
\left.\cdots+\sum_{1 \leq j_{p}<\cdots<j_{p} \leq m} \frac{d \bar{P}_{j_{1}}}{\bar{P}_{j_{1}}} \wedge \cdots \wedge \frac{d \bar{P}_{j_{p}}}{\bar{P}_{j_{p}}} \bar{\alpha}_{j_{1} \cdots j_{p}}\right\}
\end{gathered}
$$

where we may suppose that each $\bar{\alpha}_{j_{1} \ldots j_{\nu}}$ is a homogeneous polynomial $(p-\nu)$ form of degree $\mu$. Setting

$$
\begin{aligned}
\varphi=\bar{\alpha}_{0}+\sum_{j=1}^{m} \frac{d P_{j}}{P_{j}} \wedge \bar{\alpha}_{j}+ & \\
& \cdots+\sum_{1 \leq j_{1}<\cdots<j_{p}} \frac{d P_{j_{1}}}{P_{j_{1}}} \wedge \cdots \wedge \frac{d P_{j_{p}}}{P_{j_{p}}} \bar{\alpha}_{j_{1} \cdots j_{p}},
\end{aligned}
$$

we have $\sigma_{\nu}^{p}\left(\varphi \bmod F_{\mu-1} \Omega^{p}(\log D)\right)=\bar{\varphi}$.

For $p=n$, Lemma 4.3 .1 follows from $\Omega^{n}(\log D)=\frac{1}{P} \Omega^{n}\left(\mathbf{C}^{n}\right), \Omega^{n}(\log D)=$ 
$\frac{1}{\bar{P}} \Omega^{n}\left(\mathbf{C}^{n}\right)$ and the definition of the filtration $F_{\mu}$.

\section{§5. Vanishing theorem for twisted rational de Rham cohomology}

In this section we shall assume that Assumption 2.2 .1 holds for the homogeneous parts $\bar{P}_{j}$ of $P_{j}$ for $1 \leq j \leq m$.

5.1. If $\sum_{j=1}^{m} l_{j} \alpha_{j} \neq l, l-1, \ldots$, then by Theorem 3.4.1, (2), we have $H^{p}\left(\Omega^{\cdot}(\log \bar{D})_{\mu}, \nabla_{\bar{\omega}}\right)=0$ and hence by passing to long exact sequence of cohomologies of (4.3.1), we obtain

$$
H^{p-1}\left(N^{\cdot}(\log D) \mu\right) \stackrel{\sim}{\rightarrow} H^{p}\left(G r_{\mu}^{F} \Omega^{\cdot}(\log D), G r_{\mu}^{F}\left(\nabla_{\omega}\right)\right) .
$$

From Lemma 4.3.1, it follows that

$$
H^{p}\left(G r_{\mu}^{F} \Omega^{\cdot}(\log D), G r_{\mu}^{F}\left(\nabla_{\omega}\right)\right)=0 \text { for } p \neq n,
$$

which yields the following

Theorem 5.1.1. Under Assumption 2.2.1 on the homogeneous parts $\bar{P}_{j}$ of $P_{j}$, $1 \leq j \leq m$, if $\sum_{j=1}^{m} l_{j} \alpha_{j} \neq l, l-1, \ldots$, then

$$
H^{p}\left(\Omega^{\bullet}(\log D), \nabla_{\omega}\right)=0 \text { for } p \neq n
$$

Proof. Let $\varphi \in \Omega^{p}(\log D), p \neq n$ such that $\nabla_{\omega} \varphi=0$ and $\operatorname{deg} \varphi=\mu$. We denote by $[\varphi]$ the class of $\varphi$ modulo $F_{\mu-1} \Omega^{p}(\log D):[\varphi] \in G r_{\mu}^{F} \Omega^{p}(\log D)$. Since $G r_{\mu}^{F}\left(\nabla_{\omega}\right)([\varphi])=0$, by (5.1.2), we see that there exists $\phi_{\mu} \in F_{\mu} \Omega^{p-1}(\log D)$ such that

$$
\varphi_{\mu-1}:=\varphi-\nabla_{\omega} \phi_{\mu} \in F_{\mu-1} \Omega^{p}(\log D) .
$$

Notice that $F_{\mu} \Omega^{p}(\log D)=0$ for $\mu<p-l$. Since $\nabla_{\omega} \varphi_{\mu-1}=\nabla_{\omega} \varphi-\nabla_{\omega}^{2} \psi_{\mu}=0$, the similar computations apply to $\varphi_{\mu-1}$; continuing these computations, we get $\phi_{\nu} \in F_{\nu} \Omega^{p-1}(\log D)$ for $p-l \leq \nu \leq \mu$ such that

$$
\varphi-\sum_{\nu=p-l}^{\mu} \nabla_{\omega} \phi_{\nu} \in F_{p-l-1} \Omega^{p}(\log D)
$$

By the remark above, we have

$$
\varphi=\sum_{\nu=p-l}^{\mu} \nabla_{\omega} \phi_{\nu}=\nabla_{\omega}\left(\sum_{\nu=p-l}^{\mu} \phi_{\nu}\right)
$$


which shows the vanishing of $H^{p}\left(\Omega^{\bullet}(\log D), \nabla_{\omega}\right)$ for $p \neq n$.

5.2. To show the vanishing of cohomology $H^{p}\left(\Omega^{*}(* D), \nabla_{\omega}\right)$ for $p \neq n$, we first observe that $\Omega^{*}(* D)=\cup_{k=1}^{\infty} P^{-k} \Omega^{*}(\log D)$ and that $\left(P^{-k} \Omega^{*}(\log D), \nabla_{\omega}\right)$ is a subcomplex of the twisted rational de Rham complex $\left(\Omega^{*}(* D), \nabla_{\omega}\right)$. For let $\varphi=\phi / P^{k}, \phi \in \Omega^{p}(\log D)$; then by 1.2 , we have $d \phi, \frac{d P_{j}}{P_{j}} \wedge \phi \in \Omega^{p+1}(\log D)$.

On the other hand, since

$$
\nabla_{\omega} \varphi=P^{-k}\left[d \phi+\sum_{j=1}^{m}\left(\alpha_{j}-k\right) \frac{d P_{j}}{P_{j}} \wedge \phi\right],
$$

we get $\nabla_{\omega} \varphi \in P^{-k} \Omega^{p+1}(\log D)$, which means that $\left(P^{-k} \Omega^{\cdot}(\log D), \nabla_{\omega}\right)$ is a complex. We consider the isomorphism

$$
\begin{aligned}
\varepsilon_{k}^{p}: \Omega^{p}(\log D) & \longrightarrow P^{-k} \Omega^{p}(\log D) \\
\phi & \longrightarrow / P^{k}
\end{aligned}
$$

and set

$$
\omega(k):=\sum_{j=1}^{m}\left(\alpha_{j}-k\right) \frac{d P_{j}}{P_{j}}
$$

then the above computation implies that the following diagram commutes:

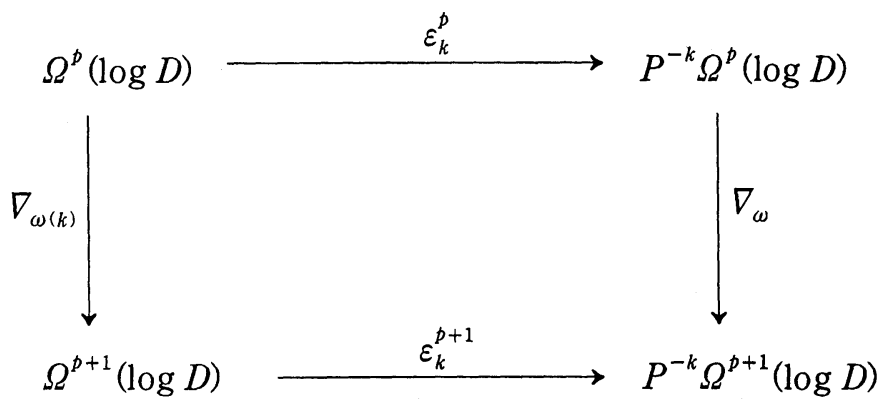

This shows that $\varepsilon_{k}^{p}, 0 \leq p \leq n$ induces an isomorphism of complexes $\dot{\varepsilon_{k}}$ : $\left(\Omega^{\bullet}(\log D), \nabla_{\omega(k)}\right) \stackrel{\sim}{\rightarrow}\left(P^{-k} \Omega^{\bullet}(\log D), \nabla_{\omega}\right)$ and hence we get

$$
H^{p}\left(\Omega^{\bullet}(\log D), \nabla_{\omega(k)}\right) \stackrel{\sim}{\rightarrow} H^{p}\left(P^{-k} \Omega^{\cdot}(\log D), \nabla_{\omega}\right) .
$$

By Theorem 5.1.1, if $\sum_{j=1}^{m} l_{j}\left(\alpha_{j}-k\right) \neq l, l-1, \ldots$, then the left hand side of (5.2.1) vanishes for $p \neq n$. Therefore if $\sum l_{j} \alpha_{j} \notin \mathbf{Z}$, then $H^{p}\left(P^{-k} \Omega^{\cdot}(\log D), \nabla_{\omega}\right)$ $=0$ for $k=1,2, \ldots$. Since $\left(\Omega^{*}(* D), \nabla_{\omega}\right)=\cup_{k=1}^{\infty}\left(P^{-k} \Omega^{*}(\log D), \nabla_{\omega}\right)$, we 
obtain the following theorem:

THEOREm 5.2.1. Suppose that Assumption 2.2.1 holds for the homogeneous part $\bar{P}_{j}$ of $P_{j}, 1 \leq j \leq m$. If $\sum_{j=1}^{m} l_{j} \alpha_{j} \notin \mathbf{Z}$, then

$$
H^{p}\left(\Omega^{\cdot}(* D), \nabla_{\omega}\right)=0 \text { for } p \neq n,
$$

and hence

$$
H^{p}\left(X, \mathscr{L}_{\omega}\right)=0 \text { for } p \neq n,
$$

where $X=\mathbf{C}^{n}-D$ and $\mathscr{L}_{\omega}$ is the complex local system consisting of horizontal sections of $\nabla_{\omega}$.

5.3. In the two dimensional case, we can show the vanishing theorem under a somewhat weaker assumption.

If $\sum l_{j} \alpha, \neq l, l-1, \ldots$, then by (5.1.1) and (5.1.2), we have

$$
\begin{gathered}
H^{0}\left(G r_{\mu}^{F} \Omega^{\cdot}(\log D), G r_{\mu}^{F}\left(\nabla_{\omega}\right)\right)=0, \\
H^{0}\left(G r_{\mu}^{F} \Omega^{\cdot}(\log D), G r_{\mu}^{F}\left(\nabla_{\omega}\right)\right) \simeq H^{0}\left(N(\log D)_{\mu}\right) .
\end{gathered}
$$

We set $\bar{P}=\bar{P}_{1} \cdots \bar{P}_{m}$ and suppose that the homogeneuos polynomial $\bar{P}$ is without multiple irreducible factors. Notice that $P=P_{1} \cdots P_{m}$ is also without multiple irreducible factors. In this case, we have $\Omega^{0}(\log D)=\Omega^{0}(\log \bar{D})=\mathbf{C}\left[u_{1}, u_{2}\right]$. In fact, let $f \in \Omega^{0}(\log D)$; then by the definition of logarithmic form, we have $a:=P f \in$ $\mathbf{C}\left[u_{1}, u_{2}\right]$ and $g_{1} d u_{1}+g_{2} d u_{2}:=\frac{d P}{P} a \in \Omega^{1}\left(\mathbf{C}^{2}\right), g_{1}, g_{2} \in \mathbf{C}\left[u_{1}, u_{2}\right]$. Notice that under our assumption, $P, \frac{\partial P}{\partial u_{i}}$ are relatively prime. Since $\frac{\partial P}{\partial u_{\imath}} a=P g_{i}$ for $i=1,2$, by the remark, we see that $P$ divides $a$. Hence from the definition of the filtration $F_{\mu}$, it follows

$$
F_{\mu} \Omega^{0}(\log D)=\{\text { polynomials of degree } \leq \mu\}
$$

Similarly we see

$$
\Omega^{0}(\log \bar{D})_{\mu}=\text { homogeneous polynomials of degree } \mu \text { \}. }
$$

Therefore we have $G r_{\mu}^{F} \Omega^{0}(\log D) \cong \Omega^{0}(\log \bar{D})_{\mu}$ and hence $N^{0}(\log D)_{\mu}=0$, which implies by (5.3.1) that

$$
H^{p}\left(G r_{\mu}^{F} \Omega^{\cdot}(\log D), G r_{\mu}^{F}\left(\nabla_{\omega}\right)\right)=0 \text { for } p=0,1 .
$$

The same reasoning as the one in 5.1 and 5.2 gives the following 
Theorem 5.3.1. Let $P_{j}\left(u_{1}, u_{2}\right)$ be polynomials in two variables $u_{1}$ and $u_{2}$. If $\sum_{j=1}^{m} l_{j} \alpha_{j} \notin \mathbf{Z}$ and the product $\bar{P}_{1} \cdots \bar{P}_{m}$ of homogeneous part $\bar{P}_{j}$ of $P_{j}, 1 \leq j \leq m$, is without multiple irreducible factor, then

$$
H^{p}\left(\Omega^{*}(* D), \nabla_{\omega}\right)=0 \text { for } p \neq 2 .
$$

Problem. Theorem 5.3.1 shows that our assumption 2.2.1 is too strong in order to prove the vanishing theorem for twisted rational de Rham cohomology. It is preferable to find some conditions which are easily checked and weaker than Assumption 2.2.1.

\section{4 .}

ExAmple. It is known (See [Ka1], [Kit1]) that Appell's hypergeometric function $F_{4}$ admits an integral representation of the following form:

$$
\int_{\sigma} \int u_{1}^{\alpha_{1}} u_{2}^{\alpha_{2}}\left(1-u_{1}-u_{2}\right)^{\alpha_{3}}\left(u_{1} u_{2}-x_{1} u_{1}-x_{2} u_{2}\right)^{\alpha_{4}} d u_{1} d u_{2}
$$

If we take $u_{1}, u_{2}, 1-u_{1}-u_{2}, u_{1} u_{2}-x_{1} u_{1}-x_{2} u_{2}$ as our $P_{1}, P_{2}, P_{3}, P_{4}$, respectively, then $\bar{P}_{1} \bar{P}_{2} \bar{P}_{3} \bar{P}_{4}=\left(u_{1} u_{2}\right)^{2}\left(-u_{1}-u_{2}\right)$ and hence it has multiple factors.

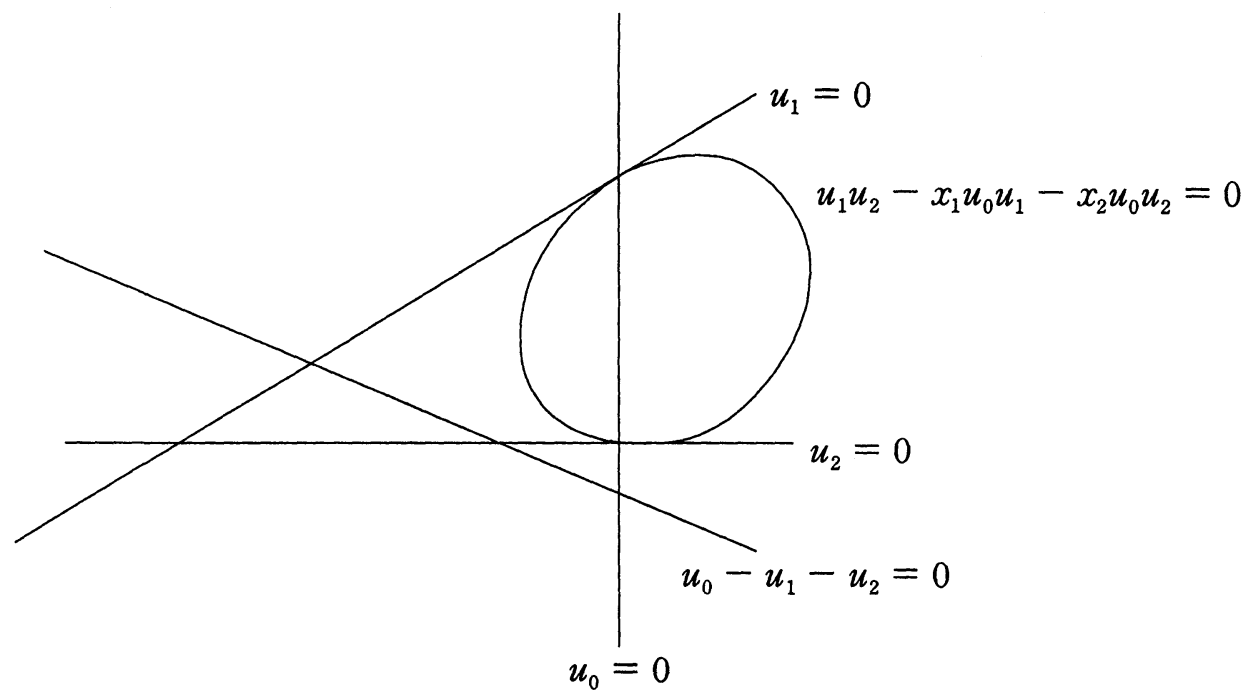

Fig. 5.1. 
If we choose the line $u_{0}-u_{1}-u_{2}=0$ as the line at infinity and make the following change of coordinates

$$
v_{1}=\frac{-u_{1}}{1-u_{1}-u_{2}}, \quad v_{2}=\frac{-u_{2}}{1-u_{1}-u_{2}}
$$

then (5.4.1) is rewritten in the following form:

$$
\iint \Pi Q_{j}\left(v_{1} v_{2}\right)^{\beta_{j}} d v_{1} \wedge d v_{2}
$$

where $Q_{1}=v_{1}, Q_{2}=v_{2}, Q_{3}=1-v_{1}-v_{2}$,

$$
\begin{gathered}
Q_{4}=x_{1} v_{1}+x_{2} v_{2}-x_{1} v_{1}^{2}+\left(1-x_{1}-x_{2}\right) v_{1} v_{2}-x_{2} v_{2}^{2}, \beta_{1}=\alpha_{1}, \beta_{2}=\alpha_{2}, \\
\beta_{3}=-\alpha_{1}-\alpha_{2}-\alpha_{3}-2 \alpha_{4}-3, \beta_{4}=\alpha_{4} .
\end{gathered}
$$

It is clear that $\Pi_{j=1}^{4} \bar{Q}_{j}$ has no multiple factors, provided $x_{1}$ and $x_{2}$ are generic in this case, by Theorem 5.3.1, the twisted cohomology $H^{p}\left(X, \mathscr{L}_{\omega}\right)$ vanishes for $p=$ 0,1 , although the condinion of Theorem 5.3.1 is not satisfied for the integral (5.4.1).

Remark. This example is due to J. Kaneko [Ka 2] who kindly communicated it to the author.

\section{§6. Arrangements, of hyperplanes in general position}

In this section we will apply our results obtained so far to arrangements of hyperplanes in general position to get some basic properties essential to the study of the hypergeometric functions of type $(n+1, m+1)$. Throughout the section we assume $m \geq n+1$.

6.1. Let

$$
P_{j}(u)=x_{0 j}+x_{1 j} u_{1}+\cdots+x_{n j} u_{n}, \quad 1 \leq j \leq m,
$$

be linear polynomials. We call the arrangement of the hyperplanes $D_{j}=\left\{P_{j}=0\right\}$, $1 \leq j \leq m$, is in general position if any $(n+1) \times(n+1)$-minor of the matrix

$$
\left(\begin{array}{cccc}
1 & x_{01} & \cdots \cdots & x_{0 m} \\
0 & x_{11} & \cdots \cdots & x_{1 m} \\
\vdots & \vdots & & \vdots \\
0 & x_{n 1} & \cdots \cdots & x_{n m}
\end{array}\right)
$$


is not zero. Then any $n \times n$ minor of the matrix

$$
\left(\begin{array}{ccc}
x_{11} & \cdots \cdots & x_{1 m} \\
\vdots & & \vdots \\
x_{n 1} & \cdots \cdots & x_{n m}
\end{array}\right)
$$

is non-zero, which means that our $\bar{P}_{1}, \ldots, \bar{P}_{m}$ satisfies Assumption 2.2 .1 and hence by Theorem 5.2.1 we obtain that if $\sum_{j=1}^{m} \alpha_{1} \notin \mathbf{Z}$, then

$$
H^{p}\left(\Omega^{\bullet}(* D), \nabla_{\omega}\right)=0 \text { for } p \neq n .
$$

6.2. By using the filtration introduced in $\S 4$, we shall study in detail the structure of $H^{p}\left(\Omega^{*}(* D), \nabla_{\omega}\right)$ to the end we prepare some lemmas below. First we extend the partial fractional decomposition to the case in several variables. Notice that since any $(n+1) \times(n+1)$-minor of $(6.1 .1)$ is non-zero, each of 1 , $u_{1}, \ldots, u_{n}$ is written as a linear combination over $\mathbf{C}$ of any $n+1$ linear polynomials of $P_{j}, 1 \leq j \leq m$. Let $g(u)=u_{1}^{\nu_{1}} \cdots u_{n}^{\nu_{n}} / P_{1} \cdots P_{m}, \nu_{\imath} \in \mathbf{Z}_{\geq 0}$, and set $|\nu|=\sum \nu_{i}$. In case $|\nu|>0$, for simplicity we suppose $\nu_{1}>0$; by the remark, we see that there exist some constants $c_{1}, \ldots, c_{n+1}$ such that

$$
u_{1}=c_{1} P_{1}+\cdots+c_{n+1} P_{n+1} \text {. }
$$

Hence

$$
g(u)=\sum_{j=1}^{n+1} c_{j} \frac{u_{1}^{\nu_{1}-1} u_{2}^{\nu_{2}} \cdots u_{n}^{\nu_{n}}}{P_{1} \cdots \hat{P}_{j} \cdots P_{m}} .
$$

In case $|\nu|=0,1$ is written as $1=\sum_{j=1}^{n+1} c_{j} P_{j}$ for some constants $c_{j} \in \mathbf{C}$ and hence

$$
g(u)=\sum_{j=1}^{n+1} c_{j} \frac{1}{P_{1} \cdots \hat{P}_{j} \cdots P_{m}} .
$$

Iterating the process, finally we arrive at the following result: if $|\nu| \geq m-n$, then

$$
\frac{u_{1}^{\nu_{1}} \cdots u_{n}^{\nu_{n}}}{P_{1} \cdots P_{m}}=\sum_{J} \frac{a_{J}(u)}{P_{j_{1}} \cdots P_{j_{n}}}
$$

where $J=\left\{j_{1}, \ldots, j_{n}\right\}, 1 \leq j_{1}<\cdots<j_{n} \leq m$ and $a_{J}(u)$ is a homogeneous polynomial of degree $|\nu|-m+n$. If $|\nu|<m-n$, then

$$
\frac{u_{1}^{\nu_{1}} \cdots u_{n}^{\nu_{n}}}{P_{1} \cdots P_{m}}=\sum_{J} \frac{c_{J}}{P_{j_{1}} \cdots P_{j_{n}}}
$$


where $J$ is as above and $c_{J} \in \mathbf{C}$.

Let $a(u)$ be a polynomial of degree $\mu$; then $a(u)$ is a linear combination of monomials $u_{1}^{\nu_{1}} \cdots u_{n}^{\nu_{n}}$. Applying the above result to each monomials, we obtain

Lemma 6.2.1. Let $a(u) \in \mathbf{C}\left[u_{1}, \ldots, u_{n}\right]$ with $\operatorname{deg} a(u)=\mu$; then the rational function $a(u) / P_{1} \cdots P_{m}$ is written as follows: if $\mu \geq m-n$, then

$$
\begin{array}{r}
\frac{a(u)}{P_{1} \cdots P_{m}}=a_{0}(u)+\sum_{j=1}^{m} a_{j}(u) \frac{1}{P_{j}}+\sum_{1 \leq j_{1}<j_{2} \leq m} a_{j_{1} j_{2}}(u) \frac{1}{P_{j_{1}} P_{j_{2}}}+ \\
\cdots+\sum_{1 \leq j_{1}<\cdots<j_{n} \leq m} a_{j_{1} \cdots j_{n}}(u) \frac{1}{P_{J_{1}} P_{J_{2}} \cdots P_{J_{n}}}
\end{array}
$$

where $a_{0}, a_{j}, a_{1_{1} j_{2}}, \ldots$ are polynomials of degree $\mu-m, \mu-m+1, \mu-m+2, \ldots$, respectively. If $\mu<m-n$, then

$$
\frac{a(u)}{P_{1} \cdots P_{m}}=\sum_{J} \frac{c_{J}}{P_{j_{1}} \cdots P_{j_{n}}}
$$

where $c_{J} \in \mathbf{C}$.

For simplicity of writing we use the following notation:

$$
x\left(\begin{array}{ccc}
i_{1} & \cdots & i_{\nu} \\
j_{1} & \cdots & j_{\nu}
\end{array}\right):=\operatorname{det}\left(\begin{array}{ccc}
x_{i_{1} j_{1}} & \cdots & x_{i_{1} j_{\nu}} \\
\vdots & & \vdots \\
x_{i_{\nu} j_{1}} & & x_{i_{\nu} j_{\nu}}
\end{array}\right) .
$$

For logarithmic $p$-forms $\varphi \in \Omega^{p}(\log D), p=n-1, n$, we will explicitly write down $\varphi$ as a sum of exterior products of $\frac{d P_{j}}{P_{j}}$ and polynomial forms (cf. Proposition 2.2.3). First let $\varphi \in \Omega^{n}(\log D)$; then $\varphi$ is written as $\varphi=$ $a(u) d u_{1} \wedge \cdots \wedge d u_{n} / P_{1} \cdots P_{m}, a(u) \in \mathbf{C}\left[u_{1}, \ldots, u_{n}\right]$. Since the rank of the matrix

$$
\left(\begin{array}{ccc}
x_{1 j_{1}} & \cdots \cdots & x_{1 j_{\nu}} \\
\vdots & & \vdots \\
x_{n j_{1}} & & x_{n j_{\nu}}
\end{array}\right)
$$

is $\nu$, there exists at least one non-zero $\nu \times \nu$-minor of the matrix which is supposed, for simplicity, to be $x\left(\begin{array}{cccc}1 & 2 & \cdots & \nu \\ j_{1} & j_{2} & \cdots & j_{\nu}\end{array}\right)$. Then from 


$$
d P_{j_{1}} \wedge \cdots \wedge d P_{j_{\nu}} \wedge d u_{\nu+1} \wedge \cdots \wedge d u_{n}=x\left(\begin{array}{ccc}
1 & \cdots & \nu \\
j_{1} & \cdots & j_{\nu}
\end{array}\right) d u_{1} \wedge \cdots \wedge d u_{n}
$$

it follows

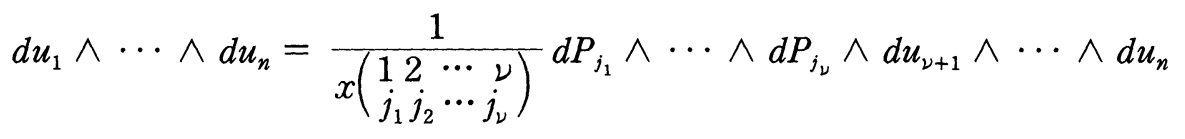

and hence

$$
\begin{aligned}
& \frac{a_{j_{1} \cdots j_{\nu}}(u)}{P_{j_{1}} \cdots P_{j_{\nu}}} d u_{1} \wedge \cdots \wedge d u_{n} \\
& =\frac{1}{x\left(\begin{array}{c}
12 \\
j_{1} j_{2} \cdots j_{\nu}
\end{array}\right)} \frac{d P_{j_{1}}}{P_{j_{1}}} \wedge \cdots \wedge \frac{d P_{j_{\nu}}}{P_{j_{\nu}}} \wedge a_{j_{1} \cdots j_{\nu}}(u) d u_{\nu+1} \wedge \cdots \wedge d u_{n} .
\end{aligned}
$$

This result, together with Lemma 6.2.1, yields the following Lemma for logarithmic $p$-forms, $p=n-1, n$.

Lemma 6.2.2 Let $\varphi \in \Omega^{p}(\log D), p=n-1, n$. Suppose $\varphi=\mu \geq 0$; then $\varphi$ is written in the form

$$
\begin{aligned}
\varphi=\varphi_{0}+\sum_{j=1}^{m} \frac{d P_{j}}{P_{j}} \wedge \varphi_{j}+\sum_{1 \leq j_{1}<j 2 \leq m} \frac{d P_{1}}{P_{j_{1}}} \wedge \frac{d P_{j_{2}}}{P_{j_{2}}} \wedge \varphi_{j_{1} j_{2}}+\cdots \\
\cdots+\sum_{1 \leq j_{1}<\cdots<j_{p} \leq m} \frac{d P_{j_{1}}}{P_{j_{1}}} \wedge \cdots \wedge \frac{d P_{j_{p}}}{P_{j_{p}}} \wedge \varphi_{j_{1} \cdots j_{p}},
\end{aligned}
$$

where $\varphi_{0}, \varphi_{j}, \ldots, \varphi_{j_{1} \ldots j_{p}}$ are polynomial forms of degree $\mu$. Moreover in case $p=n-1$, if $\operatorname{deg} \varphi=-1$, we have $\varphi=0$.

Proof. We set $* d u_{i}:=(-1)^{i-1} d u_{1} \wedge \cdots \wedge \widehat{d u_{i}} \wedge \cdots \wedge d u_{n}, 1 \leq i \leq n$. Notice that $d u_{i} \wedge * d u_{i}=d u_{1} \wedge \cdots \wedge d u_{n}$. Let $\varphi \in \Omega^{n-1}(\log D)$; then by Lemma 6.2.1, we can write $\varphi$ in the form

$$
\varphi=\sum_{i=1}^{n} \sum_{J} \frac{b_{i, J}(u)}{P_{j_{1}} \cdots P_{j_{n}}} * d u_{i}
$$

where $J=\left\{j_{1}, \ldots, j_{n}\right\}, 1 \leq j_{1}<\cdots<j_{n} \leq m$ and $b_{i, J}(u)$ is a polynomial of degree $\mu+1$. Since

$$
\frac{1}{P_{j_{1}} \cdots P_{j_{n}}} \sum_{i=1}^{n} b_{i, J}(u) * d u_{i}
$$


is a logarithmic $(n-1)$-form in a neighbourhood of $\bigcap_{\nu=1}^{n} D_{j_{\nu}}$, we have

$$
\frac{d P_{j_{\nu}}}{P_{J_{\nu}}} \wedge \sum_{i=1}^{n} b_{i, J}(u) * d u_{i} \in \Omega^{n}\left(\mathbf{C}^{n}\right) .
$$

On the other hand, since $x\left(\begin{array}{cccc}1 & 2 & \cdots & n \\ j_{1} & j_{2} & \cdots & j_{n}\end{array}\right) \neq 0$, the set $\left\{d P_{j_{1}} \wedge \cdots \wedge \widehat{d P_{J_{\nu}}} \wedge \cdots\right.$ $\left.\wedge d P_{j_{n}}\right\}_{1 \leq \nu \leq n}$ forms a basis of $\Lambda^{n-1} T^{*} X$ at each point of $X$ and by the general theory of exterior algebra, the base change from $\left\{* d u_{i}\right\}_{1 \leq \nu \leq n}$ to $\left\{d P_{j_{1}} \wedge \cdots \wedge\right.$ $\left.\widehat{d P} \jmath_{\nu} \wedge \cdots \wedge d P_{j_{n}}\right\}_{1 \leq \nu \leq n}$ is given by the matrix of which entries are the $(n-1) \times(n-1)$ minors of the matrix

$$
\left(\begin{array}{ccc}
x_{1 j_{1}} & \cdots \cdots & x_{1 j_{n}} \\
\vdots & & \vdots \\
x_{n j_{1}} & \cdots \cdots & x_{n j_{n}}
\end{array}\right) .
$$

Therefore $\sum b_{i, J} * d u_{i}$ is expressible in the form

$$
\sum_{\nu=1}^{n} c_{j_{\nu}}(u) d P_{j_{1}} \wedge \cdots \wedge \widehat{d P_{j_{\nu}}} \wedge \cdots \wedge d P_{j_{n}}, c_{j_{\nu}} \in \mathbf{C}\left[u_{1}, \ldots, u_{n}\right], \operatorname{deg} c_{j_{\nu}}=\mu+1 ;
$$

which, together with (6.2.3), yields

$$
\frac{1}{P_{j_{\nu}}} c_{j_{\nu}}(u) d P_{j_{1}} \wedge \cdots \wedge d P_{j_{n}} \in \Omega^{n}\left(\mathbf{C}^{n}\right) .
$$

This implies that $P_{j_{\nu}}$ divides $c_{j_{\nu}}$. Notice that if $\mu=-1$, this is equivalent to $c_{j_{\nu}}=$ 0 and hence $\varphi=0$. We set $c_{j_{\nu}}=P_{j_{\nu}} \tilde{c}_{j_{\nu}}(u)$; then we have

$$
\varphi=\sum_{J} \sum_{\nu=1}^{n} \frac{P_{j_{\nu}} \tilde{c}_{j_{\nu}}}{P_{j_{1}} \cdots P_{j_{n}}} * d P_{j_{\nu}}, \tilde{c}_{j_{\nu}} \in \mathbf{C}\left[u_{1}, \ldots, u_{n}\right] \text { with } \operatorname{deg} \tilde{c}_{j_{\nu}}=\mu,
$$

where we use the abbreviation $* d P_{j_{\nu}}=d P_{j_{1}} \wedge \cdots \wedge \widehat{d P_{j_{\nu}}} \wedge \cdots \wedge d P_{j_{n}}$. By simple computations, it is shown that $\varphi$ is written in the form (6.2.2).

Remark. By Lemma 6.2.1 and the proof of Lemma 6.2.2, we see easily that for any $\varphi \in \Omega^{p}(\log D), \varphi$ is expressible in the form (6.2.2). But in general we cannot conclude that the $\varphi_{\rho_{1} \ldots \rho_{\nu}}$ 's are of degree $\mu$.

6.3. For the comparison of $H^{n}\left(\Omega^{*}(\log D), \nabla_{\omega}\right)$ with $H^{n}\left(\Omega^{*}(* D), \nabla_{\omega}\right)$, we will prove here the reduction theorem of rational forms to logarithmic forms. 
Lemma 6.3.1. We assume $\alpha_{j} \notin \mathbf{Z}_{>0}$ for $1 \leq j \leq m$ and $p=n-1$ or $n$. Let $\varphi$ be in $\Omega^{p}(* D)$ such that $\nabla_{\omega} \varphi \in \frac{1}{P_{1} \cdots P_{m}} \Omega^{p+1}\left(\mathbf{C}^{n}\right)$; then we can write $\varphi$ in the form

$\varphi=\phi+\nabla_{\omega} \beta$

where $\phi \in \Omega^{p}(\log D)$ and $\beta \in \Omega^{p-1}(* D)$.

Proof. $\varphi$ is expressible as

$$
\varphi=\tilde{\varphi} / P_{1}^{k_{1}} \cdots P_{m}^{k_{m}}, \quad \tilde{\varphi} \in \Omega^{p}\left(\mathbf{C}^{n}\right), \quad k_{j} \geq 1 \quad \text { for } \quad 1 \leq j \leq m .
$$

It suffices to show that if $k_{j}>1$, then we can find $\alpha \in \Omega^{p}\left(\mathbf{C}^{n}\right)$ and $\beta \in \Omega^{n-1}(* D)$ such that

$$
\varphi=\alpha /\left(P_{1}^{k_{1}} \cdots P_{j}^{k_{j}-1} \cdots P_{m}^{k_{m}}\right)+\nabla_{\omega} \beta
$$

For since $\nabla_{\omega}\left(\alpha /\left(P_{1}^{k_{1}} \cdots P_{j}^{k_{j}-1} \cdots P_{m}^{k_{m}}\right)\right)$ is in $\frac{1}{P} \Omega^{p+1}\left(\mathbf{C}^{n}\right)$, we can iterate these computations to obtain that $\varphi$ is written in the form $\varphi=\frac{\alpha}{P_{1} \cdots P_{m}}+\nabla_{\omega} \beta$ for some $\alpha \in \Omega^{p}\left(\mathbf{C}^{n}\right)$ and $\beta \in \Omega^{p-1}(* D)$. By $\nabla_{\omega}\left(\alpha /\left(P_{1} \cdots P_{m}\right)\right) \in \frac{1}{P} \Omega^{p+1}\left(\mathbf{C}^{n}\right)$ and Lemma 1.3.1, we can conclude that $\alpha / P_{1} \cdots P_{m} \in \Omega^{p}(\log D)$.

Since

$$
\nabla_{\omega} \varphi=\frac{1}{\Pi P_{j}^{k_{j}}}\left\{d \tilde{\varphi}+\sum_{j=1}^{m}\left(\alpha_{j}-k_{j}\right) \frac{d P_{j}}{P_{j}} \wedge \tilde{\varphi}\right\} \in \frac{1}{P_{1} \cdots P_{m}} \Omega^{p+1}\left(\mathbf{C}^{n}\right)
$$

and each $k_{j} \geq 1$, we have

$$
d \tilde{\varphi}+\sum\left(\alpha_{j}-k_{j}\right) \frac{d P_{j}}{P_{j}} \wedge \tilde{\varphi} \in \Omega^{n+1}\left(\mathbf{C}^{n}\right) .
$$

By assumption, $P_{1}$ and $P_{j}$ are relatively prime and $\alpha_{j}-k_{j} \neq 0$; hence $\frac{d P_{j}}{P_{j}} \wedge \tilde{\varphi}$ $\in \Omega^{p+1}\left(\mathbf{C}^{n}\right)$, which shows that $\tilde{\varphi} / P_{1} \cdots P_{m}$ is in $\Omega^{p}(\log D)$. By the remark at the and of $6.2, \tilde{\varphi} / P_{1} \cdots P_{m}$ is expressible in the following form

$$
\begin{aligned}
\tilde{\varphi}=P_{1} \cdots P_{m}\left\{\varphi_{0}+\sum \frac{d P_{j}}{P_{j}}\right. & \wedge \tilde{\varphi}_{j}+\cdots+ \\
& \left.+\sum_{1 \leq j_{1}<\cdots<j_{p}} \frac{d P_{j_{1}}}{P_{j_{1}}} \wedge \cdots \wedge \frac{d P_{j_{p}}}{P_{j_{p}}} \tilde{\varphi}_{j_{1} \cdots j_{p}}\right\},
\end{aligned}
$$




$$
\tilde{\varphi}_{j_{1} \cdots j_{\nu}} \in \Omega^{p-\nu}\left(\mathbf{C}^{n}\right) .
$$

Hence $\varphi$ is written as a sum of the following terms:

$$
[\tilde{\varphi}]_{J} / P_{1}^{k_{1}} \cdots P_{m}^{k_{m}}
$$

where we set $J=\left\{j_{1}, \ldots, j_{\nu}\right\}, 1 \leq j_{1}<\cdots<j_{\nu} \leq m$, and

$$
[\hat{\varphi}]_{J}:=P_{1} \cdots P_{m} \frac{d P_{j_{1}}}{P_{j_{1}}} \wedge \cdots \wedge \frac{d P_{j_{\nu}}}{P_{j_{\nu}}} \wedge \hat{\varphi}_{j_{1} \cdots j_{\nu}}
$$

For simplicity, we suppose $k_{1}>1$ and divide two cases.

First case: If $1 \notin\left\{j_{1}, \ldots, j_{\nu}\right\}=J$, then $[\tilde{\varphi}]_{J}$ is expressible as $P_{1} \times$ polynomial $p$-forms and hence the term (6.3.1) reduces to the form (polynomial $p$-form)/ $P_{1}^{k_{1}-1} P_{2}^{k_{2}} \cdots P_{m}^{k_{m}}$

Second case where $1 \in J$. For simplicity we assume $J=\{1, \ldots, \nu\}$; then

$$
[\tilde{\varphi}]_{J}=d P_{1} \wedge \cdots \wedge d P_{\nu} \wedge\left(P_{\nu+1} \cdots P_{m} \tilde{\varphi}_{J}\right)
$$

Set

$$
\xi=d P_{2} \wedge \cdots \wedge d P_{\nu} \wedge\left(P_{\nu+1} \cdots P_{m} \tilde{\varphi}_{J}\right) / P_{1}^{k_{1}-1} P_{2}^{k_{2}} \cdots P_{m}^{k_{m}}
$$

then simple computations give

$$
\begin{aligned}
& \nabla_{\omega} \xi=\left(\alpha_{1}-k_{1}+1\right) \frac{[\tilde{\varphi}]_{J}}{P_{1}^{k_{1}} \cdots P_{m}^{k_{m}}}+(-1)^{\nu-1} \frac{d P_{2} \wedge \cdots \wedge d P_{\nu} \wedge d\left(P_{\nu+1} \cdots P_{m} \tilde{\varphi}_{J}\right)}{P_{1}^{k_{1}-1} P_{2}^{k_{2}} \cdots P_{m}^{k_{m}}} \\
&+\sum_{j=\nu+1}^{m}\left(\alpha_{j}-k_{j}\right) \frac{d P_{j}}{P_{j}} \wedge \frac{d P_{2} \wedge \cdots \wedge d P_{\nu} \wedge\left(P_{\nu+1} \cdots P_{m} \tilde{\varphi}_{J}\right)}{P_{1}^{k_{1}-1} P_{2}^{k_{2}} \cdots P_{m}^{k_{m}}}
\end{aligned}
$$

The sum of the second and third terms in the right hand side of (6.3.2) is written as $\eta / P_{1}^{k_{1}-1} P_{2}^{k_{2}} \cdots P_{m}^{k_{m}}$ where $\eta \in \Omega^{p}\left(\mathbf{C}^{n}\right)$. Since $\alpha_{1} \notin \mathbf{Z}_{>0}$, we have

$$
\frac{[\tilde{\varphi}]_{J}}{P_{1}^{k_{1}} \cdots P_{m}^{k_{m}}}=\frac{1}{\alpha_{1}-k_{1}+1} \frac{\eta}{P_{1}^{k_{1}-1} P_{2}^{k_{2}} \cdots P_{m}^{k_{m}}}+\nabla_{\omega}\left(\frac{\xi}{\alpha_{1}-k_{1}+1}\right) .
$$

This completes the proof of Lemma 6.3.1.

6.4. Let $j:\left(\Omega^{*}(\log D), \nabla_{\omega}\right) \rightarrow\left(\Omega^{*}(* D), \nabla_{\omega}\right)$ be the natural inclusion of complexes. We shall show that the induced homomorphism $j_{*}: H^{p}\left(\Omega^{*}(\log D)\right.$, $\left.\nabla_{\omega}\right) \rightarrow H^{p}\left(\Omega^{\cdot}(* D), \nabla_{\omega}\right)$ is an isomorphism for all $p$ under the assumptions $\alpha_{j} \notin$ $\mathbf{Z}_{>0}$ for $1 \leq j \leq m$ and $\sum_{j=1}^{m} \alpha_{j} \notin \mathbf{Z}$. The statement holds for $0 \leq p \leq n-1$ by 
Theorem 5.1.1 and Theorem 5.2.1. To show it for $p=n$, let $\varphi \in \Omega^{n}(* D)$; since $\nabla_{\omega} \varphi=0$, by Lemma 6.3.1, $\varphi$ is expressible as $\varphi=\phi+\nabla_{\omega} \beta, \phi \in \Omega^{n}(\log D)$, $\beta \in \Omega^{n-1}(* D)$, which shows $j_{*}$ is surjective. To show $j_{*}$ is injective, let $\phi \in$ $\Omega^{n}(\log D)$ such that $\phi=\nabla_{\omega} \varphi$ for some $\varphi \in \Omega^{n-1}(* D)$ Since

$$
\phi \in \frac{1}{P_{1} \cdots P_{m}} \Omega^{n}\left(\mathbf{C}^{n}\right)
$$

again by Lemma 6.3.1, $\varphi$ is expressible as $\varphi=\alpha+\nabla_{\omega} \beta, \alpha \in \Omega^{n-1}(\log D), \beta \in$ $\Omega^{n-2}(* D)$ and hence $\phi=\nabla_{\omega} \alpha$, which shows that $\psi$ is zero in $H^{n}\left(\Omega^{*}(\log D)\right.$, $\nabla_{\omega}$ ) and hence $j_{*}$ is injective. Thus we have

THEOREM 6.4.1. In case of arrangement of hyperplanes in general position, if $\alpha_{j} \notin \mathbf{Z}_{>0}$ for $1 \leq j \leq m$ and $\sum \alpha_{j} \notin \mathbf{Z}$, then

$$
\begin{aligned}
& H^{p}\left(\Omega^{\cdot}(* D), \nabla_{\omega}\right)=0 \text { for } p \neq n, \\
& H^{n}\left(\Omega^{\cdot}(* D), \nabla_{\omega}\right) \simeq H^{n}\left(\Omega^{\cdot}(\log D), \nabla_{\omega}\right) .
\end{aligned}
$$

6.5. By investigating the filtration $F$. on $\Omega^{n-1}(\log D)$ in detail, we will determine the structure of $H^{n}\left(\Omega^{\circ}(\log D), \nabla_{\omega}\right)$. We have shown in 5.1 that if $\sum \alpha_{j} \neq m, m-1, \ldots$, then

$$
H^{n}\left(G r_{\mu}^{F} \Omega^{\cdot}(\log D), G r_{\mu}^{F}\left(\nabla_{\omega}\right)\right) \simeq H^{n-1}\left(N^{\cdot}(\log D)_{\mu}\right)
$$

where $N^{n-1}(\log D)_{\mu}$ is defined in 4.3 by the cokernel of the mapping

$$
\sigma_{\mu}^{n-1}: G r_{\mu}^{F} \Omega^{n-1}(\log D) \rightarrow \Omega^{n-1}(\log \bar{D})_{\mu} .
$$

For simplicity of writing, we use the following notations:

$\Omega^{p}\left(\mathbf{C}^{n}\right)_{\nu}$ : space of homogeneous polynomial $p$-forms of degree $\nu$,

$\Omega^{p}\left(\mathbf{C}^{n}\right)_{\leq \nu}$ : space of polynomial $p$-form of degree $\leq \nu$,

$$
\varphi\left\langle j_{1}, \ldots, j_{p}\right\rangle:=\frac{d P_{j_{1}}}{P_{j_{1}}} \wedge \cdots \wedge \frac{d P_{j_{p}}}{P_{j_{p}}} .
$$

Notice that a polynomial $p$-form is of degree $\geq p$. By the remark and Lemma 6.2.2, $\xi \in F_{1} \Omega^{n-1}(\log D)$ is expressible in the form

$$
\xi=\sum \varphi\left\langle j_{1} \cdots j_{n-1}\right\rangle \xi_{j_{1} \cdots j_{n-1}}+\sum \varphi\left\langle j_{1} \cdots j_{n-2}\right\rangle \wedge \xi_{j_{1} \cdots j_{n-2}}
$$

where $\xi_{j_{1} \cdots j_{n-1}} \in \Omega^{0}\left(\left(\mathbf{C}^{n}\right)_{\leq 1}\right.$ and $\xi_{j_{1} \ldots j_{n-2}} \in \Omega^{1}\left(\mathbf{C}^{n}\right)_{\leq 1}$. In the above expression, each summand $\varphi\langle\nu\rangle \wedge \xi_{J}, J=\left\{j_{1}, \ldots, j_{p}\right\}, p=n-1$ or $n$, belongs to $F_{1} \Omega^{n-1}(\log D)$ and hence we can write $F_{1} \Omega^{n-1}(\log D)$ in the following form: 


$$
\begin{aligned}
F_{1} \Omega^{n-1}(\log D)=\sum \varphi\left\langle j_{1},\right. & \left.\ldots, j_{n-1}\right\rangle \wedge \Omega^{0}\left(\mathbf{C}^{n}\right)_{\leq 1}+ \\
& +\sum \varphi\left\langle j_{1}, \ldots, j_{n-2}\right\rangle \wedge \Omega^{1}\left(\mathbf{C}^{n}\right)_{\leq 1} .
\end{aligned}
$$

We can show the similar results for $F_{\mu} \Omega^{n-1}(\log D)$ which are summarized in the following table: Here we abbreviate the space

$$
\sum_{1 \leq j_{1}<\cdots<j_{p} \leq m} \varphi\left\langle j_{1}, \ldots, j_{p}\right\rangle \wedge \Omega^{n-1-p}\left(\mathbf{C}^{n}\right)_{\leq \nu}
$$

as $[p] \wedge \Omega_{\leq \nu}^{n-1-p}$ and so on.

\begin{tabular}{|c|c|c|c|c|c}
$F_{-1}$ & $F_{0}$ & $F_{1}$ & $F_{2}$ & $\cdots$ & $F_{\mu}$ \\
\hline 0 & {$[n-1] \wedge \Omega_{0}^{0}$} & {$[n-1] \wedge \Omega_{\leq 1}^{0}$} & {$[n-1] \wedge \Omega_{\leq 2}^{0}$} & $\cdots$ & {$[n-1] \wedge \Omega_{\leq \mu}^{0}$} \\
\hline & {$[n-2] \wedge \Omega_{1}^{1}$} & {$[n-2] \wedge \Omega_{\leq 2}^{1}$} & $\cdots \cdots$ & {$[n-2] \wedge \Omega_{\leq \mu}^{1}$} \\
\hline & {$[n-3] \wedge \Omega_{2}^{2}$} & $\cdots$ & {$[n-3] \wedge \Omega_{\leq \mu}^{2}$} \\
\hline & & {$[n-4] \wedge \Omega_{\leq \mu}^{3}$} \\
\cline { 4 - 5 }
\end{tabular}

Table 1.

6.6. Now we study the structure of $\Omega^{n-1}(\log \bar{D})$; since our arrangement is, by assumption, hyperplanes in general position, each of $u_{1}, \ldots, u_{n}$ is written as a linear combination over $\mathbf{C}$ of any $n$ linear homogeneous polynomials of $\bar{P}_{j}, 1 \leq j$ $\leq m$. Investigating in more detail the proof of Proposition 2.2.3 in this case, we obtain the following

Lemma 6.6.1. Let $\bar{\varphi} \in \Omega^{n-1}(\log \bar{D})_{\mu}$ with $\mu \geq 0$; then $\bar{\varphi}$ can be written in the form

$$
\begin{aligned}
\bar{\varphi}= & \bar{\varphi}_{0}+\sum_{j=1}^{m} \frac{d \bar{P}_{j}}{\bar{P}_{j}} \wedge \bar{\varphi}_{j}+\cdots \\
& \cdots+\sum_{1 \leq j_{1}<\cdots<j_{n-1} \leq m} \frac{d \bar{P}_{j_{1}}}{\bar{P}_{j_{1}}} \wedge \cdots \wedge \frac{d \bar{P}_{j_{n-1}}}{\bar{P}_{j_{n-1}}} \cdot \bar{\varphi}_{j_{1} \cdots j_{n-1}}
\end{aligned}
$$

where $\bar{\varphi}_{j_{1} \ldots j_{\nu}} \in \Omega^{n-1-\nu}\left(\mathbf{C}^{n}\right)_{\mu}$.

Proof. Since we can prove Lemma 6.6.1 along the same line of the proof of Proposition 2.2.3, we will sketch the outline of the proof. Let $\bar{\varphi} \in \Omega^{n-1}(\log \bar{D})_{\mu}$ with $\mu \geq 0$; then we can write $\bar{\varphi}$ as $\bar{\varphi}=\phi / \bar{P}_{1} \cdots \bar{P}_{m}$ where $\phi$ is a homogeneous 
polynomial $(n-1)$-form of degree $\mu+m$. We have, by definition,

$$
\frac{d \bar{P}_{j}}{\bar{P}_{j}} \wedge \phi \in \Omega^{n}(\mathbf{C})
$$

and then

$$
d \bar{P}_{j} \wedge \phi \equiv 0\left(\bmod \bar{P}_{j}\right) \text { for } 1 \leq j \leq m .
$$

By induction on $m$, we show that $\phi$ is written in the form

$$
\begin{aligned}
\phi= & \bar{P}_{1} \cdots \bar{P}_{m}\left\{\psi_{0}+\sum_{j=1}^{m} \frac{d \bar{P}_{j}}{\bar{P}_{j}} \wedge \phi_{j}+\cdots\right. \\
& \left.\cdots+\sum_{1 \leq j_{1}<\cdots<j_{n-1} \leq m} \frac{d \bar{P}_{j_{1}}}{\bar{P}_{j_{1}}} \wedge \cdots \wedge \frac{d \bar{P}_{j_{n-1}}}{\bar{P}_{j_{n-1}}} \psi_{j_{1} \cdots j_{n-1}}\right\}
\end{aligned}
$$

where $\phi_{j_{1} \ldots j_{n}}$ is homogeneous of degree $\mu$.

In case $m=1$, we can write $\phi$ as

$$
\phi=\sum_{i=1}^{n} a_{i}(u) * d \bar{P}_{i}, \quad a_{i}(u) \in \Omega^{0}\left(\mathbf{C}^{n}\right)_{\mu+m-n+1}
$$

since $\bar{P}_{1}, \ldots, \bar{P}_{n}$ form a system of coordinates of $\mathbf{C}^{n}$. By (6.6.2), we get $d \bar{P}_{1} \wedge \phi=$ $a_{1}(u) * 1 \equiv 0\left(\bmod \bar{P}_{1}\right)$ where $* 1=d \bar{P}_{1} \wedge \cdots \wedge d \bar{P}_{n}$ and hence $\bar{P}_{1}$ divides $a_{1}(u): a_{1}(u)=\bar{P}_{1} \tilde{a}_{1}(u)$. This, together with (6.6.4), implies that $\phi$ is written in the form (6.6.3).

We suppose the statement is true for $m$ and show that it is also true for $m+$ 1. Let $\phi \in \Omega^{n-1}\left(\mathbf{C}^{n}\right)_{\mu+m+1}$ such that

$$
d \bar{P}_{j} \wedge \phi \equiv 0\left(\bmod \bar{P}_{j}\right) \text { for } 1 \leq j \leq m+1 .
$$

Then by induction assumption, $\phi$ is written in the form (6.6.3) where $\phi_{j_{1} \ldots j_{\nu}}$ is homogeneous of degree $\mu+1$. We will show the statement by induction on the largest number $N$ for which $\phi_{j_{1} \cdots j_{N}} \neq 0$. If $N \leq n-2$, then the proof of Proposition 2.2 .3 works in this case and hence the statement is true for $N \leq n-2$. In case $N=n-1$, first notice that $\phi_{j_{1} \cdots j_{n-1}}$ is a homogeneous polynomial of degree $\mu+1: \operatorname{deg} \phi_{j_{1} \ldots j_{n-1}} \geq 1$. Since $\bar{P}_{j_{1}}, \ldots \bar{P}_{j_{n-1}}, \bar{P}_{m+1}$ form a system of coordinates of $\mathbf{C}^{n}$, by the remark, we can write $\phi_{j_{1} \cdots j_{n-1}}$ as

$$
\phi_{j_{1} \cdots j_{n-1}}=\sum_{k=1}^{n-1} \bar{P}_{j_{k}} \alpha_{j_{1} \cdots j_{n-1} ; j_{k}}+\bar{P}_{m+1} \alpha_{j_{1} \cdots j_{n-1} ; m+1} .
$$

Substitute (6.6.5) into (6.6.3); then from the same reasoning as in the proof of 
Proposition 2.2 .3 (in (2.2.6), set $\beta_{\beta_{1} \cdots j_{n-1} ; j_{k}}=\beta_{j_{1} \cdots j_{n-1} ; m+1}=0$ and apply the reasoning to our case) it follows that the statement is true for $N=n-1$. This completes induction.

As in 6.5 , we set

$$
[\bar{p}] \wedge \Omega_{\nu}^{n-1-p}=\sum_{1 \leq j_{1}<\cdots<j_{p} \leq m} \frac{d \bar{P}_{j_{1}}}{\bar{P}_{j_{1}}} \wedge \cdots \wedge \frac{d \bar{P}_{j_{p}}}{\bar{P}_{j_{p}}} \wedge \Omega^{n-1-p}\left(\mathbf{C}^{n}\right)_{\nu} ;
$$

then as in 6.5 , we have

LEMma 6.6.2. We have

$$
\begin{aligned}
\Omega^{n-1}(\log \bar{D})_{0}=[\overline{n-1}] & \wedge \Omega_{0}^{0}, \\
\Omega^{n-1}(\log \bar{D})_{1}=[\overline{n-1}] & \wedge \Omega_{1}^{0}+[\overline{n-2}] \wedge \Omega_{1}^{1}, \\
& \cdots \cdots \cdots \\
\Omega^{n-1}(\log \bar{D})_{\mu}=[\overline{n-1}] & \wedge \Omega_{\mu}^{0}+[\overline{n-2}] \wedge \Omega_{\mu}^{1}+\cdots .
\end{aligned}
$$

From Table 1 and Lemma 6.6.2 it follows that if $\mu \geq 0$, then the mapping $\sigma_{\mu}^{n-1}: G r_{\mu}^{F} \Omega^{n-1}(\log D) \rightarrow \Omega^{n-1}(\log \bar{D})_{\mu}$ is surjective and hence $N^{n-1}(\log D)_{\mu}=0$ for $\mu \geq 0$. Therefore by $(6.5 .1)$ we have $H^{n}\left(G r_{\mu}^{F} \Omega^{*}(\log D), G r_{\mu}^{F}\left(\nabla_{\omega}\right)\right)=0$ for $\mu \geq 0$. Repeating the reasoning in the proof of Theorem 5.1.1, we conclude that any $\varphi \in \Omega^{n}(\log D)$ is cohomologous to a $\tilde{\varphi}$ in $F_{-1} \Omega^{n}(\log D)$.

Since

$$
F_{-1} \Omega^{n}(\log D)=\frac{d u_{1} \wedge \cdots \wedge d u_{n}}{P_{1} \cdots P_{m}} \Omega_{\leq m-n-1}^{0} \quad \text { and } F_{-1} \Omega^{n-1}(\log D)=0,
$$

we have an isomorphism

$$
H^{n}\left(\Omega^{\cdot}(\log D), \nabla_{\omega}\right) \simeq \frac{d u_{1} \wedge \cdots \wedge d u_{n}}{P_{1} \cdots P_{m}} \Omega_{\leq m-n-1}^{0}
$$

Notice that $\operatorname{dim} \Omega_{\leq m-n-1}^{0}=\left(\begin{array}{c}m-1 \\ n\end{array}\right)$. We summarize the results in

THEOREM 6.6.3. In case of arrangement of hyperplanes in general position, if $\alpha_{j} \notin \mathbf{Z}_{>0}$ for $1 \leq j \leq m$ and $\sum_{j=1}^{m} \alpha_{j} \notin \mathbf{Z}$, then

$$
\begin{aligned}
& H^{p}\left(\Omega^{*}(* D), \nabla_{\omega}\right)=0 \text { for } p \neq n, \\
& H^{n}\left(\Omega^{\cdot}(* D), \nabla_{\omega}\right) \simeq \\
& \quad\left\{\frac{a(u)}{P_{1} \cdots P_{m}} d u_{1} \wedge \cdots \wedge d u_{n} \mid a(u) \in \mathbf{C}[u], \operatorname{deg} a \leq m-n-1\right\}
\end{aligned}
$$


and

$$
\operatorname{dim} H^{n}\left(\Omega^{\cdot}(* D), \nabla_{\omega}\right)=\left(\begin{array}{c}
m-1 \\
n
\end{array}\right)
$$

Since $F_{0} \Omega^{n-1}(\log D)$ is the space spanned by $\varphi\left\langle j_{1}, \ldots, j_{n-1}\right\rangle$, we have also the following isomorphism

$$
H^{n}\left(\Omega^{\cdot}(* D), \nabla_{\omega}\right) \simeq \frac{\left\{\left\{\varphi\left\langle j_{1}, \ldots, j_{n}\right\rangle \mid 1 \leq j_{1}<\cdots<j_{n} \leq m\right\}\right\}}{\omega \wedge\left\{\left\{\varphi\left\langle j_{1}, \ldots, j_{n-1}\right\rangle \mid 1 \leq j_{1}<\cdots<j_{n-1} \leq m\right\}\right\}}
$$

COROLlary We can choose

$$
\frac{d P_{j_{1}}}{P_{j_{1}}} \wedge \cdots \wedge \frac{d P_{j_{n}}}{P_{j_{n}}}, 1 \leq j_{1}<\cdots<j_{n} \leq m-1,
$$

as a basis of $H^{n}\left(\Omega^{*}(* D), \nabla_{\omega}\right)$.

Proof. It suffices to observe that

$$
\omega \wedge \varphi\left\langle j_{1}, \ldots, j_{n-1}\right\rangle=\sum_{k=1}^{m} \alpha_{k} \varphi\left\langle k, j_{1}, \ldots, j_{n-1}\right\rangle
$$

and hence

$$
\varphi\left\langle m, j_{1}, \ldots, j_{n-1}\right\rangle=-\sum_{k=1}^{m-1} \frac{\alpha_{k}}{\alpha_{m}} \varphi\left\langle k, j_{1}, \ldots, j_{n-1}\right\rangle
$$

in $H^{n}\left(\Omega^{\cdot}(* D), \nabla_{\omega}\right)$.

\section{REFERENCES}

[Ao] K. Aomoto, Les équations aux différences linéaires et les intégrales des fonctions multiformes, J. Fac. Sci. Univ. Tokyo, Sec. IA. 22 (1975), 271-297 and: Une correction et un complément à l'article "Les équations aux différences linéaires et les intégrales des fonctions multiformes", ibid., 26 (1979), $519-523$.

[AKOT] K. Aomoto, M. Kita, P. Orlik and H. Terao, On the structure of twisted de Rham cohomology groups associated to hypergeometric integrals, preprint (1993).

[deR] G. de Rham, Sur la division de formes et de courants par une formes linéaire, Comment. Math. Helv., 28 (1954), 346-352.

[Ka1] J. Kaneko, Monodromy group of Apell's system $\left(F_{4}\right)$, Tokyo J. Math., 4 (1981), $35-54$.

[Ka2] - Private communication, (1981). 
[Kit1] M. Kita, On hypergeometric functions in several variables 1, New integral representations of Euler type, Japan. J. Math., 18 (1992), 25-74.

[Kit2] - On hypergeometric functions in several variables 2 , The Wronskian of the hypergeometric functions of type $(n+1, m+1)$, J. Math. Soc. Japan., 45 (1993), 645-669.

[K-N] M. Kita and M. Noumi, On the structure of cohomology groups attached to the integral of certain many-valued analytic functions, Japan. J. Math., 9 (1983), $113-157$.

[M] H. Matsumura, Commutative algebra, W. A. Benjamin Co., New York (1980).

[MSY] K. Matsumoto, T. Sasaki and M. Yoshida, The monodromy of the period map of a 4-parameter family of K3-surfaces and the hypergeometric function of type (3.6), Internat. J. Math., 3 (1992), 1-164.

[O-T] P. Orlik and H. Terao, Arrangements and Milnor fibres, preprint (1993).

[R-T] L. Rose and H. Terao, A free resolution of the module of logarithmic forms of a generic arrangement, J. Algebra, 136 (1991), 376-400.

[S1] K. Saito, Theory of logarithmic differential forms and logarithmic vector fields, J. Fac. Sci. Univ. Tokyo, Sec. IA, 27 (1980), 265-291.

[S2] - On a generalization of de Rham lemma, Ann. Inst. Fourier, Grenoble, 26 (1976), 165-170.

[Ya] T. Yamazaki, Private communication, (1981).

College of Liberal Arts

Kanazawa University

Kakuma

Kanazawa 920-11, Japan 